\title{
Formation of Synthetically Versatile 2-Aminobenzophenones from Readily Accessed Acyl Hydrazides
}

\author{
Nehaal Ahmed, André Shamsabadi, and Vijay Chudasama*i) \\ Department of Chemistry, University College London, 20 Gordon Street, London WC1H 0AJ, U.K. \\ Supporting Information
}

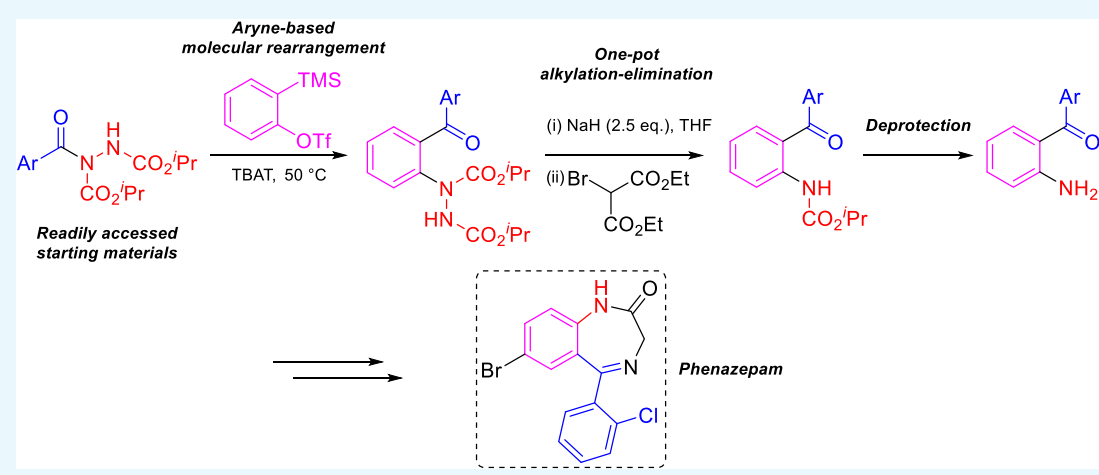

ABSTRACT: Herein, we report the transformation of readily accessed acyl hydrazides into protected 2-aminobenzophenones via a two-step process involving an aryne-based molecular rearrangement followed by a one-pot addition-elimination procedure. The assembly of the scaffold is tolerant of a wide variety of functional groups, and the carbamate group on the product can be facilely removed to afford highly valuable 2-aminobenzophenones. Application of the protocol was demonstrated in the synthesis of neurological medicine phenazepam.

\section{INTRODUCTION}

2-Aminobenzophenones are a very important class of compounds in medicinal and organic chemistry. Compounds bearing this structure have displayed desirable pharmacological use as antimitotic, ${ }^{1}$ antitumor, ${ }^{2}$ and antiproliferative ${ }^{3}$ agents, as well as skeletal muscle relaxants. ${ }^{4}$-Aminobenzophenones are also useful starting materials for the synthesis of a wide variety of fine chemicals such as acridones, ${ }^{5}$ quinolines, ${ }^{6-8}$ quinazolines, ${ }^{9-11}$ quinolinones, ${ }^{12}$ quinoxalinones, ${ }^{13}$ fluorenones, ${ }^{14}$ benzisoxazoles, ${ }^{15}$ indazoles, ${ }^{16}$ indoles, ${ }^{17}$ 2-quinazolinones, ${ }^{18}$ benzothiophenes, ${ }^{19}$ diaryldibenzodiazocines, ${ }^{20}$ and (perhaps most noteworthy) benzodiazepines. ${ }^{21-24}$ As such, various methodologies for the synthesis of 2-aminobenzophenones and their derivatives have been developed, including (i) Friedel-Crafts acylation of para-substituted anilines; ${ }^{25,26}$ (ii) reaction of 2-aminobenzaldehydes with aryl Grignard reagents followed by oxidation with $\mathrm{CuCl}_{2} ;{ }^{27}$ (iii) Pd-catalyzed addition of arylboronic acids ${ }^{28}$ or sodium arylsulfinates ${ }^{29}$ to 2 -aminobenzonitriles; (iv) Pd-catalyzed $\mathrm{C}-\mathrm{H}$ bond coupling of orthodirected anilines; ${ }^{30}$ and (v) aryl insertion into the $\mathrm{C}-\mathrm{N}$ of amides $^{31}$ or imides. ${ }^{32}$ Unfortunately, however, many of these processes suffer from either poor substrate scope (e.g., FriedelCrafts processes falter when utilizing electron-poor arenes), require multiple reaction steps (e.g., the Grignard-based route obviates the presence of acidic protons and requires product oxidation to obtain the ketone), or require the use of expensive metal catalysts (e.g., $\left.\mathrm{Pd}(\mathrm{OAc})_{2}, \mathrm{Pd}(\mathrm{TFA})_{2}\right)$. Application of aryne insertion chemistry appears to be the most promising of these conditions; however, work here has been primarily focused on the formation of $\mathrm{N}$-aryl 2-aminobenzophenones. ${ }^{31}$

There has been a substantial number of recent reports for the synthesis of acyl hydrazides and their conversion into a variety of useful chemical functionalities. ${ }^{33}$ More specifically, facilely accessed acyl hydrazides have been reported as shelf-stable intermediates for the creation of esters, thioesters, amides, ${ }^{34}$ ketones, ${ }^{35} \mathrm{~N}$-acyl carbamates, ${ }^{36} 1 \mathrm{H}$ - and $2 \mathrm{H}$-indazoles, ${ }^{37}$ and $1,3,4$-oxadiazoles, ${ }^{38}$ as well as being employed as precursors for the formation of bioactive molecules such as hydroxamic acids ${ }^{39}$ and macrocyclic enamides. ${ }^{40}$ Recently, we developed conditions for the reaction of arynes with acyl hydrazides in which a novel molecular rearrangement reaction pathway facilitates the formation of 2-hydrazobenzophenones. ${ }^{37}$ We envisioned that the transformation of this entity into useful 2-aminobenzophenones would be possible via rupture of the $\mathrm{N}-\mathrm{N}$ bond in a onepot alkylation-E1cB elimination procedure (Scheme 1). The cleavage of the $\mathrm{N}-\mathrm{N}$ bond without the use of hydrogenation procedures involving expensive/undesirable metals was seen as a key aspect in itself; this is particularly well described in related work by Magnus et al. ${ }^{41}$ Finally, we postulated that our methodology could be applied for the synthesis of medicinally relevant benzodiazepines.

Received: October 14, 2019

Accepted: November 27, 2019

Published: December 17, 2019 


\section{Scheme 1. Synthetic Routes To Access 2-Aminobenzophenones}

Yan (2011)

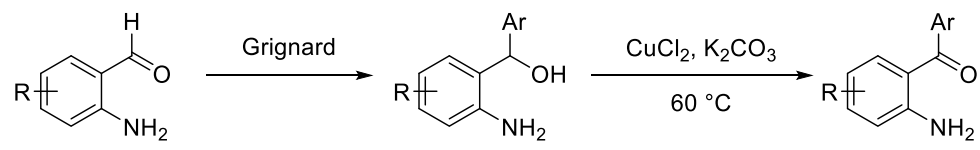

Cortez-Maya (2012)

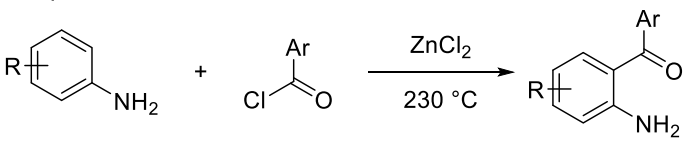

Chen (2014)

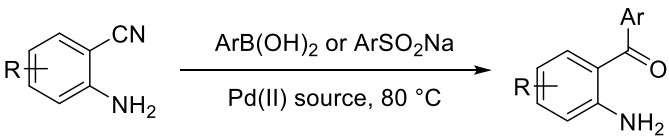

This work

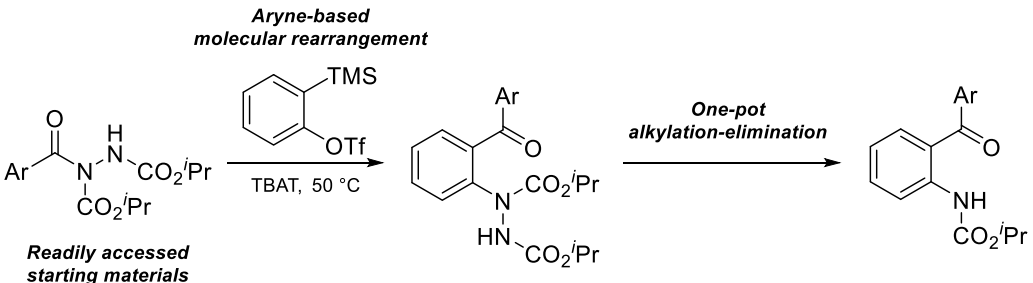

\section{RESULTS AND DISCUSSION}

Our study began with the reaction of 2-hydrazobenzophenone $\mathbf{1}$ a with tert-butyl bromoacetate $\mathbf{2 a}$ in the presence of a base with the aim of forming protected 2 -aminobenzophenone $3 \mathbf{a}$ in a two-step one-pot procedure. Initially, the reaction was carried out using an excess of $\mathrm{NaH}$ at $20^{\circ} \mathrm{C}$ (Table 1 , entry 1$) .{ }^{36}$ While

Table 1. Reaction Optimization for the Formation of Protected 2-Aminobenzophenone 3a

$\begin{array}{clllcccc}\text { entry } & \text { base } & \text { equiv } & \begin{array}{c}T \\ \left({ }^{\circ} \mathrm{C}\right)\end{array} & \begin{array}{c}\text { alkylating } \\ \text { agent }\end{array} & \begin{array}{c}\text { yield 3a } \\ \text { equiv }\end{array} & \begin{array}{c}\text { yield 4 } \\ (\%)\end{array} \\ 1 & \mathrm{NaH} & 5 & 20 & \mathbf{2 a} & 1.1 & 45(72) & 0 \\ 2 & \mathrm{NaH} & 5 & 50 & \mathbf{2 a} & 1.1 & 67 & 0 \\ 3 & \mathrm{NaH} & 3 & 50 & \mathbf{2 a} & 1.1 & 60 & 12 \\ 4^{b} & \mathrm{NaH} & 1+1 & 20 & \mathbf{2 a} & 1.1 & 77 & 0 \\ 5^{c} & \mathrm{NaH} & 1 & 20 & \mathbf{2 b} & 1.1 & 35(55) & 0 \\ 6^{d} & \mathrm{NaH} & 2 & 20 & \mathbf{2 b} & 2 & 57 & 10 \\ 7^{d} & \mathrm{NaH} & 2.5 & 20 & \mathbf{2 b} & 2.5 & 53 & 0 \\ 8^{e} & \mathrm{NaH} & 2.5 & 20 & \mathbf{2 b} & 2.5 & 82 & 0 \\ 9 & \mathrm{Cs}_{2} \mathrm{CO}_{3} & 2.5 & 20 & \mathbf{2 b} & 2.5 & 20(90) & 19 \\ 10 & \mathrm{NEt}_{3} & 2.5 & 20 & \mathbf{2 b} & 2.5 & 0(2) & 0 \\ 11 & \mathrm{NaO}^{t} \mathrm{Bu} & 2.5 & 20 & \mathbf{2 b} & 2.5 & 23(44) & 0 \\ 12 & \mathrm{NaOEt} \mathrm{b} & 2.5 & 20 & \mathbf{2 b} & 2.5 & 42(77) & 0\end{array}$

${ }^{a}$ Conversion of starting material 1a given in parentheses when not $100 \%$. ${ }^{b}$ Stepwise procedure with intermediate $4 a$ isolated. ${ }^{c} 45 \%$ of starting material recovered. ${ }^{d}$ Malonate salt observed. ${ }^{e}$ Dropwise addition of alkylating agent.

these conditions did provide access to desired product 3a, it was only in a modest yield of $45 \%$. Increasing the temperature to 50 ${ }^{\circ} \mathrm{C}$ had a positive effect on yield with $67 \%$ of $3 \mathrm{a}$ being isolated (Table 1, entry 2 ).

Unfortunately, the efficiency of the reaction was not improved by lowering the number of equivalents of base to 3 , where a $60 \%$ yield of the desired product and a $12 \%$ yield of alkylatedhydrazide intermediate $4 \mathbf{a}$ were recovered (Table 1 , entry 3 ).
We next decided to try a two-step process, i.e., the use of 1 equiv of $\mathrm{NaH}$ and 1.1 equiv of tert-butyl bromoacetate $2 \mathrm{a}$ at room temperature to isolate the resulting alkylated acyl hydrazide intermediate $\mathbf{4 a}$ and then subjecting this intermediate to an additional equivalent of $\mathrm{NaH}$ relative to intermediate isolated. This resulted in an improved overall isolated yield, 77\%, of desired product 3 a over the two steps (Table 1, entry 4). In an effort to further improve the efficiency of the process, we utilized an alkylating agent with two electron-withdrawing groups in an attempt to promote elimination. We first utilized 1.1 equiv of diethyl bromomalonate $\mathbf{2} \mathbf{b}$ as the alkylating agent with 1 equiv of $\mathrm{NaH}$ in an attempt to isolate alkylated intermediate $\mathbf{4 b}$ (Table 1 , entry 5). Interestingly, no intermediate was isolated under these conditions with a $35 \%$ yield of the protected 2-aminobenzophenone product $3 \mathrm{a}$ and $45 \%$ recovery of starting material 1a observed. We suspected that the increased acidity of the $\alpha$ proton in alkylated-hydrazide intermediate $4 \mathrm{~b}$ resulted in rapid elimination to product 3a. In an attempt to exploit this, a onepot procedure using 2 equiv of base and alkylating agent was trialed; however, this resulted in only a $57 \%$ yield of 3 a due to the formation of a significant amount of a malonate salt (Table 1 , entry 6). Increasing the equivalents of $\mathrm{NaH}$ and malonate $\mathbf{2 b}$ to 2.5 resulted in a slight decrease in yield (53\%) with even more malonate salt observed (Table 1 , entry 7 ). We therefore added malonate $\mathbf{2} \mathbf{b}$ dropwise to minimize the consumption of $\mathrm{NaH}$ prior to intermediate $\mathbf{4 b}$ formation. Gratifyingly, this resulted in a high yield of the desired product, $82 \%$ (Table 1, entry 8 ), with no intermediate or malonate salt observed. Having established a procedure that led to the formation of $\mathbf{3 a}$ in excellent yield, the suitability of other bases was appraised (Table 1, entries 9-12). Unfortunately, the substitution of $\mathrm{NaH}$ for weaker bases (i.e., $\mathrm{Cs}_{2} \mathrm{CO}_{3}, \mathrm{NEt}_{3}, \mathrm{NaO}^{t} \mathrm{Bu}, \mathrm{NaOEt}$ ) proved detrimental to yield with triethylamine not even facilitating the initial alkylation step. The other bases were able to carry out the alkylation and E1cB elimination steps, but gave low yields of the desired product. In the case of using $\mathrm{Cs}_{2} \mathrm{CO}_{3}$, we highlight that although 
Scheme 2. Reaction Scope for the Formation of Protected 2-Aminobenzophenones $3^{b}$

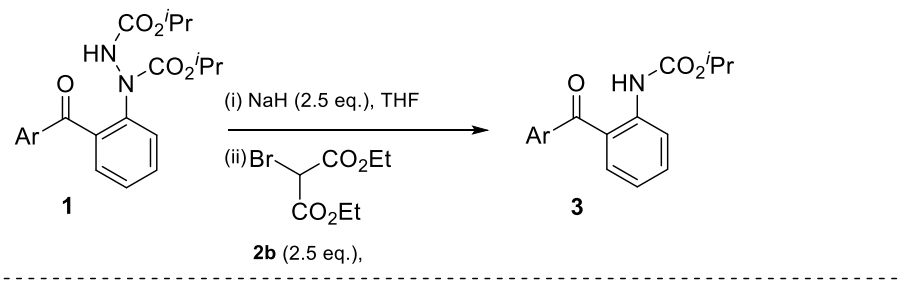

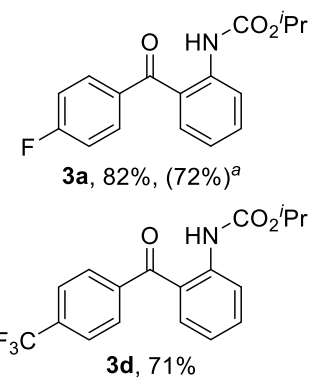

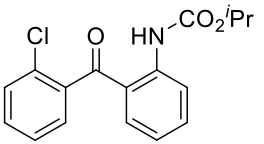

$3 g, 71 \%$<smiles>CCCC(=O)Nc1ccccc1C(=O)c1ccccc1</smiles>

3b, $78 \%$<smiles>COc1ccc(C(=O)c2ccccc2NC(=O)OC(C)C)cc1</smiles><smiles>COc1ccc(C(=O)c2ccccc2NC(=O)OC(C)C)c(OC)c1OC</smiles>

$3 \mathrm{~h}, 72 \%$<smiles>CCCOC(=O)Nc1ccccc1C(=O)c1cccc(Br)c1</smiles>

$3 c, 68 \%$<smiles>CCCOC(=O)Nc1ccccc1C(=O)c1cccc(Br)c1</smiles><smiles>CCCOC(=O)Nc1ccccc1C(=O)c1ccc(C)c(C)c1</smiles><smiles>CCCOC(=O)Nc1ccccc1C(=O)c1cccc(I)c1</smiles>

$3 \mathbf{j}, 68 \%$

${ }^{a}$ Gram scale. ${ }^{b}$ Reaction conditions: 2 -aminobenzophenone 1 ( $0.50 \mathrm{mmol}, 1$ equiv) with NaH ( $1.25 \mathrm{mmol}, 2.5$ equiv) in tetrahydrofuran (THF) ( 3 $\mathrm{mL})$ followed by the dropwise addition of diethyl bromomalonate $2 \mathrm{~b}\left(1.25 \mathrm{mmol}, 2.50\right.$ equiv) at $20{ }^{\circ} \mathrm{C}$ for $4 \mathrm{~h}$.

compounds $\mathbf{3 a}$ and $\mathbf{4 b}$ were isolated, the crude reaction mixture appeared to be a complex mixture of many products.

With the optimized conditions for the transformation of 2hydrazobenzophenones into protected 2 -aminobenzophenones in hand, we took the opportunity to investigate the applicability of our protocol for the formation of various protected 2aminobenzophenones (Scheme 2). A range of 2-hydrazobenzophenones were examined $(\mathbf{1} \mathbf{a}-\mathbf{1 j})$ under the developed reaction conditions. All of the starting 2-hydrazobenzophenones were prepared in good yields using our previously reported procedure for the reaction of acyl hydrazides with benzynes (see the Supporting Information for details).

To our delight, the reaction was tolerant of various functional groups on the aromatic motif (e.g., halo, methyl, methoxy, and trifluoromethyl functionalities) with good to excellent yields (64-82\%) observed. The reaction protocol proved to be tolerant of electron-poor, electron-neutral, and electron-rich aromatic rings, with ortho-, meta-, and para-substitutions having no significant effect on yield. The optimized protocol was also shown to be applicable to a gram-scale synthesis, with compound 3a being prepared in a $72 \%$ yield.

Having established an efficient protocol for the formation of protected 2-aminobenzophenones, we next investigated conditions for deprotection. Initially, we trialed previously reported Brønsted-acid-based conditions ${ }^{42}$ and observed that refluxing $3 \mathbf{b}$ in $\mathrm{HCl}(12 \mathrm{M})$ resulted in the full conversion of starting material with an excellent yield of 2 -aminobenzophenone $\mathbf{5 b}$ obtained (Table 2, entries 1 and 2). Other previously reported conditions, the use of aqueous $\mathrm{KOH}$ in dimethylacetamide, ${ }^{43}$ did not result in the full conversion of starting material; even
Table 2. Reaction Optimization for the Formation of 2Aminobenzophenone $5 b^{a}$

\begin{tabular}{clllcc} 
entry & \multicolumn{1}{c}{ reagent } & solvent & \multicolumn{1}{c}{$T\left({ }^{\circ} \mathrm{C}\right)$} & $\begin{array}{c}\text { conversion } 3 \mathbf{b} \\
(\%)\end{array}$ & $\begin{array}{c}\text { yield } \mathbf{5 b} \\
(\%)\end{array}$ \\
1 & $\mathrm{HCl}(12 \mathrm{M})$ & dioxane & 110 & 100 & 88 \\
2 & $\mathrm{HCl}(12 \mathrm{M})$ & $\mathrm{EtOAc}$ & 110 & 100 & 83 \\
3 & $\mathrm{KOH}(0.3 \mathrm{M})$ & $\mathrm{DMA}$ & 60 & 26 & 22 \\
4 & $\mathrm{KOH}(0.5 \mathrm{M})$ & $\mathrm{DMA}$ & 60 & 42 & 30 \\
5 & $\mathrm{KOH}(1 \mathrm{M})$ & $\mathrm{DMA}$ & 110 & 51 & 38 \\
6 & $\mathrm{KOH}(4 \mathrm{M})$ & $\mathrm{DMA}$ & 110 & 67 & 59 \\
7 & $\mathrm{KOH}(8 \mathrm{M})$ & $\mathrm{DMA}$ & 110 & 56 & 54 \\
8 & $\mathrm{AlCl}_{3}$ & DCM & $0(45 \mathrm{~min})$ & 100 & 92
\end{tabular}

${ }^{a}$ DMA $=N, N$-dimethylacetamide. $\mathrm{DCM}=$ dichloromethane. All reactions carried out over $16 \mathrm{~h}$ unless stated otherwise in parentheses.

increasing the reaction temperature and concentration of $\mathrm{KOH}$ did not improve the yield and conversion significantly (Table 2, entries 3-7). Most pleasingly, however, the use of Lewis acid $\mathrm{AlCl}_{3}$ in dichloromethane resulted in the rapid deprotection (45 $\mathrm{min}$ ) of the isopropyl carbamate with an exceptional isolated yield of product $\mathbf{5 b}$ observed, $92 \%$ (Table 2, entry 8 ; a $60 \%$ overall yield of $\mathbf{5 b}$ from its corresponding acyl hydrazide). ${ }^{44}$ The assertion of Lewis acidity having a role in the reaction is based on the fact that the use of $\mathrm{HCl}(12 \mathrm{M})$ alone (in either 1,4-dioxane or EtOAc) only resulted in complete conversion after $16 \mathrm{~h}$ at 110 ${ }^{\circ} \mathrm{C}$, i.e., rather than being complete in $45 \mathrm{~min}$ at $0{ }^{\circ} \mathrm{C}$ when using $\mathrm{AlCl}_{3}$. In fact, the reaction when using $\mathrm{HCl}(12 \mathrm{M})$ after $16 \mathrm{~h}$ at room temperature only resulted in an $11 \%$ yield of $\mathbf{5 b}(17 \%$ conversion of $\mathbf{3 b}$ ). The optimized deprotection conditions were 
Scheme 3. Synthesis of Phenazepam ${ }^{a}$

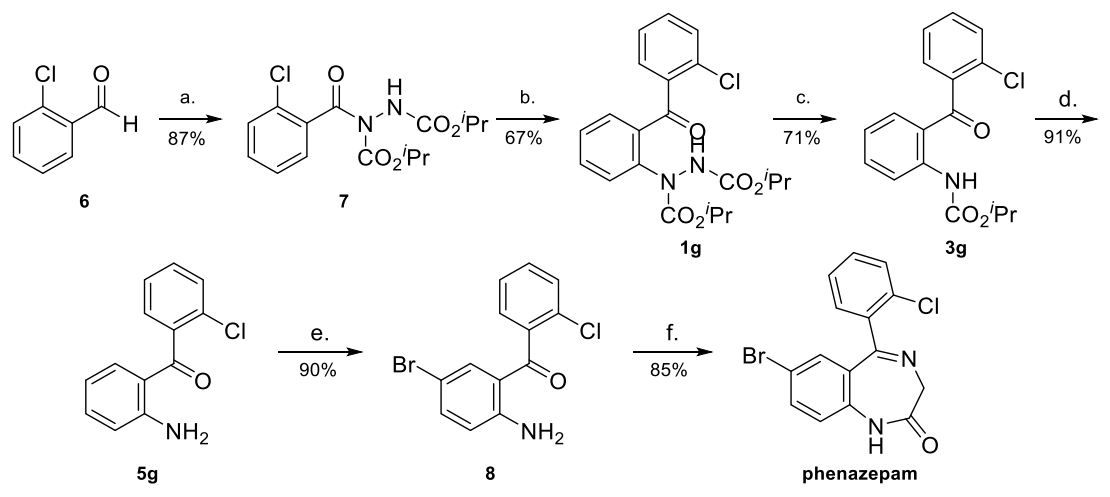

${ }^{a}$ Reagents and conditions: (a) DIAD (1.1 equiv), $\mathrm{H}_{2} \mathrm{O}, \mathrm{rt}, 24 \mathrm{~h}$; (b) 2-(trimethylsilyl)phenyl trifluoromethanesulfonate (1.5 equiv), TBAT (2 equiv), toluene, $60^{\circ} \mathrm{C}, 16 \mathrm{~h}$; (c) $\mathrm{NaH}$ (2.5 equiv), THF, diethyl bromomalonate (2.5 equiv, dropwise addition), rt, $4 \mathrm{~h}$; (d) $\mathrm{AlCl}_{3}(4 \mathrm{equiv}), \mathrm{DCM}$, rt, $45 \mathrm{~min}$; (e) NBS ( 1 equiv), DCM, $0{ }^{\circ} \mathrm{C}, 1 \mathrm{~h}$; (f) bromoacetyl bromide (1.1 equiv), DCM, $0{ }^{\circ} \mathrm{C}, 1 \mathrm{~h}$, followed by $\mathrm{NH}_{3}, \mathrm{EtOH}, \mathrm{rt}, 24 \mathrm{~h}$. TBAT = tetrabutylammonium difluorotriphenylsilicate.

then trialed on electron-poor and electron-rich protected 2aminobenzophenones $\mathbf{3 d}$ and $\mathbf{3 h}$ (respectively). To our delight, this afforded the corresponding 2-aminobenzophenones $\mathbf{5 d}$ and Sh in excellent yields ( $>85 \%$, Table 2 , inset).

Recognizing the potential of the protocol for the synthesis of functional 2-aminobenzophenones, we turned our attention to providing a tangible application of the protocol for the synthesis of a pharmaceutically relevant benzodiazepine (Scheme 3), as a representative example, especially as aryne chemistry is being increasingly employed to synthesize such systems. ${ }^{45}$ Phenazepam is a member of a class of molecules that have found use in the treatment of various psychiatric and neurological disorders. ${ }^{46}$ Furthermore, phenazepam can be employed as a precursor for the formation of more complex benzodiazepines such as cinazepam and 3-hydroxyphenazepam. ${ }^{47}$ We envisioned that phenazepam could be readily accessed via our optimized methodology. More specifically, the chloro entity could be incorporated through the use of starting aldehyde 6 and the bromo substituent could be installed through para-directed bromination of 2-aminobenzophenone 8. Initially, 2-chlorobenzaldehyde 6 was transformed into the corresponding acyl hydrazide 7 via hydroacylation of diisopropyl azodicarboxylate $(\mathrm{DIAD})$. Aryl insertion into the $\mathrm{C}(\mathrm{O})-\mathrm{N}$ bond utilizing arynebased molecular transformation resulted in 2-hydrazobenzophenone $1 \mathrm{~g}$ in a $67 \%$ yield. This species was submitted to our addition-elimination protocol to afford protected 2-aminobenzophenone $3 \mathrm{~g}$ in a $71 \%$ yield. Lewis acidic deprotection of the carbamate group afforded 2-aminobenzophenone $\mathbf{5 g}$ in a $91 \%$ yield. para-Directed electrophilic bromination using NBS introduced bromine at the 7-position to form 8 in a $90 \%$ yield. Finally, a one-pot alkylation-cyclization procedure led to an excellent yield of phenazepam (85\%). As such, through the application of our novel methodology, we were able to obtain the desired benzodiazepine in good yield (and even without any of the reaction steps being optimized).

\section{CONCLUSIONS}

In conclusion, we have shown 2-hydrazobenzophenones to be excellent candidates for the synthesis of functional 2-aminobenzophenones, which have many applications in terms of their pharmacological and synthetic uses (as exemplified by the extensive catalogue of bioactive molecules they can be transformed into). ${ }^{5-24}$ A one-pot, alkylation-E1cB elimination procedure to produce isopropyl carbamate protected 2-aminobenzophenones from 2-hydrazobenzophenones has been developed. Moreover, the protocol is tolerant of many functional groups, and the deprotection of the isopropyl carbamate has been optimized to produce 2-aminobenzophenones in high yield. Finally, the application of both of these methods was demonstrated in the synthesis of phenazepam.

\section{EXPERIMENTAL SECTION}

General Experimental Methods. All reagents were purchased from Sigma-Aldrich or AlfaAesar and were used as received without further purification unless otherwise stated. Where described below, petrol refers to petroleum ether (b.p. $\left.40-60{ }^{\circ} \mathrm{C}\right)$. All reactions were monitored by thin-layer chromatography (TLC) on precoated silica gel plates. Silica get plates were initially examined under short-wave UV light and then developed with the use of an aqueous potassium permanganate stain. Flash column chromatography was carried out with preloaded GraceResolv flash cartridges on a Biotage Isolera Spektra One flash chromatography system. Quoted yields refer to chromatographically and spectroscopically pure compounds unless otherwise stated. ${ }^{1} \mathrm{H}$ NMR spectra were recorded at 300,600 , or $700 \mathrm{MHz}$ and ${ }^{13} \mathrm{C} \mathrm{NMR}$ at 150 or 175 $\mathrm{MHz}$ on a Bruker Avance 300, Bruker Avance III 600, or Bruker Avance Neo 700 spectrometer. All spectra were recorded at room temperature unless stated otherwise. The chemical shifts $(\delta)$ for ${ }^{1} \mathrm{H}$ and ${ }^{13} \mathrm{C}$ are quoted relative to residual signals of the solvent on the parts per million (ppm) scale. Coupling constants ( $J$ values) are reported in hertz $(\mathrm{Hz})$ and are reported as $J_{\mathrm{H}-\mathrm{H}}$ unless otherwise stated. Signal multiplicities in ${ }^{13} \mathrm{C}$ NMR were determined using the distortionless enhancement by polarization transfer (DEPT) spectral editing technique. Melting points were measured with a Gallenkamp apparatus and are uncorrected.

General Experimental for the Formation of Acyl Hydrazides: Method A. To a solution of azodicarboxylate (6.00 mmol, 1.2 equiv) in $\mathrm{H}_{2} \mathrm{O}(1 \mathrm{~mL})$ was added aldehyde ( $5.00 \mathrm{mmol}, 1.0$ equiv), and the reaction mixture was stirred at $21^{\circ} \mathrm{C}$ for $48 \mathrm{~h}$. The resulting solution was extracted with EtOAc $(3 \times 15 \mathrm{~mL})$. The combined extracts were dried $\left(\mathrm{MgSO}_{4}\right)$ and filtered, and the solvent was evaporated in vacuo. The resultant crude residue was purified as described below. 
Diisopropyl 1-(4-Fluorobenzoyl)hydrazine-1,2-dicarboxylate. Compound prepared according to method A. Purification by column chromatography (10-50\% EtOAc/petrol) afforded diisopropyl 1-(4-fluorobenzoyl)hydrazine-1,2-dicarboxylate as a white solid (1.21 g, $3.70 \mathrm{mmol}, 74 \%) .{ }^{1} \mathrm{H}$ NMR (600 MHz, $\left.\mathrm{CDCl}_{3}\right) \delta 7.80-7.62(\mathrm{~m}, 2 \mathrm{H}), 7.11(\mathrm{t}, J=8.5 \mathrm{~Hz}, 2 \mathrm{H}), 6.95-$ $6.70(\mathrm{~m}, \mathrm{NH}, 1 \mathrm{H}), 5.02$ (septet, $J=6.1 \mathrm{~Hz}, 1 \mathrm{H}), 4.92$ (septet, $J=$ $6.1 \mathrm{~Hz}, 1 \mathrm{H}), 1.30(\mathrm{~d}, J=5.2 \mathrm{~Hz}, 6 \mathrm{H}), 1.12(\mathrm{~d}, J=5.0 \mathrm{~Hz}, 6 \mathrm{H})$; ${ }^{13} \mathrm{C} \mathrm{NMR}\left(150 \mathrm{MHz}, \mathrm{CDCl}_{3}\right) \delta 170.3(\mathrm{C}), 165.2\left(\mathrm{~d}, \mathrm{~J}_{\mathrm{C}-\mathrm{F}}=\right.$ $253.4 \mathrm{~Hz}, \mathrm{C}$ ), 155.4 (C), 152.9 (C), $131.3\left(\mathrm{~d}, J_{\mathrm{C}-\mathrm{F}}=2.7 \mathrm{~Hz}\right.$, $\mathrm{CH}), 131.1\left(\mathrm{~d}, J_{\mathrm{C}-\mathrm{F}}=9.8 \mathrm{~Hz}, \mathrm{CH}\right), 115.5\left(\mathrm{~d}, J_{\mathrm{C}-\mathrm{F}}=22.1 \mathrm{~Hz}\right.$, $\mathrm{CH}), 72.8(\mathrm{CH}), 70.9(\mathrm{CH}), 22.0\left(\mathrm{CH}_{3}\right), 21.5\left(\mathrm{CH}_{3}\right)$; IR (solid) 3306, 2984, 2939, 1704, 1602, $1507 \mathrm{~cm}^{-1}$.

Diisopropyl 1-Benzoylhydrazine-1,2-dicarboxylate. Compound prepared according to method A. Purification by column chromatography (10-50\% EtOAc/petrol) afforded diisopropyl 1-benzoylhydrazine-1,2-dicarboxylate as a white solid (1.02 g, $3.30 \mathrm{mmol}, 66 \%) .{ }^{1} \mathrm{H}$ NMR $\left(600 \mathrm{MHz}, \mathrm{CDCl}_{3}\right) \delta 7.72-7.58$ $(\mathrm{m}, 2 \mathrm{H}), 7.50(\mathrm{t}, J=7.4 \mathrm{~Hz}, 1 \mathrm{H}), 7.40(\mathrm{t}, J=7.6 \mathrm{~Hz}, 2 \mathrm{H}), 7.15-$ $6.99(\mathrm{~m}, \mathrm{NH}, 1 \mathrm{H}), 5.00$ (septet, $J=6.3 \mathrm{~Hz}, 1 \mathrm{H}), 4.87$ (septet, $J=$ $5.9 \mathrm{~Hz}, 1 \mathrm{H}), 1.28$ (d, $J=5.5 \mathrm{~Hz}, 6 \mathrm{H}), 1.04(\mathrm{~d}, J=4.7 \mathrm{~Hz}, 6 \mathrm{H})$; ${ }^{13} \mathrm{C} \mathrm{NMR}\left(150 \mathrm{MHz}, \mathrm{CDCl}_{3}\right) \delta 171.4(\mathrm{C}), 155.5(\mathrm{C}), 153.0$ (C), $135.3(\mathrm{CH}), 132.0(\mathrm{CH}), 128.2(\mathrm{CH}), 72.6(\mathrm{CH}), 70.7$ (CH), $22.0\left(\mathrm{CH}_{3}\right), 21.4\left(\mathrm{CH}_{3}\right)$; IR (solid) 3308, 2983, 2938, $1705,1601 \mathrm{~cm}^{-1}$.

Diisopropyl 1-(3-Bromobenzoyl)hydrazine-1,2-dicarboxylate. Compound prepared according to method A. Purification by column chromatography (10-50\% EtOAc/petrol) afforded diisopropyl 1-(3-bromobenzoyl)hydrazine-1,2-dicarboxylate as a white solid (1.20 g, $3.10 \mathrm{mmol}, 62 \%) .{ }^{1} \mathrm{H}$ NMR $(600 \mathrm{MHz}$, $\left.\mathrm{CDCl}_{3}\right) \delta 7.84-7.73(\mathrm{~m}, 1 \mathrm{H}), 7.67-7.52(\mathrm{~m}, 2 \mathrm{H}), 7.30(\mathrm{t}, J=$ $7.8 \mathrm{~Hz}, 1 \mathrm{H}), 6.99-6.80(\mathrm{~m}, \mathrm{NH}, 1 \mathrm{H}), 5.01$ (septet, $J=6.0 \mathrm{~Hz}$, $1 \mathrm{H}), 4.91$ (septet, $J=6.0 \mathrm{~Hz}, 1 \mathrm{H}), 1.30(\mathrm{~d}, J=5.8 \mathrm{~Hz}, 6 \mathrm{H}), 1.10$ $(\mathrm{d}, J=5.1 \mathrm{~Hz}, 6 \mathrm{H}) ;{ }^{13} \mathrm{C} \mathrm{NMR}\left(150 \mathrm{MHz}, \mathrm{CDCl}_{3}\right) \delta 169.8(\mathrm{C})$, $155.3(\mathrm{C}), 152.6(\mathrm{C}), 137.2(\mathrm{C}), 134.8(\mathrm{CH}), 131.0(\mathrm{CH})$, $129.9(\mathrm{CH}), 126.7(\mathrm{CH}), 122.2(\mathrm{C}), 73.0(\mathrm{CH}), 71.0(\mathrm{CH})$, $22.0\left(\mathrm{CH}_{3}\right), 21.5\left(\mathrm{CH}_{3}\right)$; IR (solid) 3303, 2983, 2938, 1707, $1568 \mathrm{~cm}^{-1}$.

Diisopropyl 1-(4-(Trifluoromethyl)benzoyl)hydrazine-1,2dicarboxylate. Compound prepared according to method A. Purification by column chromatography (10-50\% EtOAc/ petrol) afforded diisopropyl 1-(4-(trifluoromethyl)benzoyl)hydrazine-1,2-dicarboxylate as a white solid $(1.17 \mathrm{~g}, 3.10 \mathrm{mmol}$, $62 \%) .{ }^{1} \mathrm{H}$ NMR $\left(600 \mathrm{MHz}, \mathrm{CDCl}_{3}\right) \delta 7.82-7.74(\mathrm{~m}, 2 \mathrm{H})$, $7.73-7.67(\mathrm{~m}, 2 \mathrm{H}), 6.95-6.70(\mathrm{~m}, \mathrm{NH}, 1 \mathrm{H}), 5.03$ (septet, $J=$ $6.1 \mathrm{~Hz}, 1 \mathrm{H}), 4.91$ (septet, $J=6.1 \mathrm{~Hz}, 1 \mathrm{H}), 1.31(\mathrm{~d}, J=5.8 \mathrm{~Hz}$, $6 \mathrm{H}), 1.10(\mathrm{~d}, J=5.1 \mathrm{~Hz}, 6 \mathrm{H}) ;{ }^{13} \mathrm{C} \mathrm{NMR}\left(150 \mathrm{MHz}, \mathrm{CDCl}_{3}\right) \delta$ 170.2 (C), $155.3(\mathrm{C}), 152.6$ (C), 138.8 (C), 133.4 (q, $J_{\mathrm{C}-\mathrm{F}}=$ $31.9 \mathrm{~Hz}, \mathrm{C}), 128.3(\mathrm{CH}), 125.3\left(\mathrm{q}, J_{\mathrm{C}-\mathrm{F}}=3.0 \mathrm{~Hz}, \mathrm{CH}\right), 123.7$ $\left(\mathrm{q}, J_{\mathrm{C}-\mathrm{F}}=272.6 \mathrm{~Hz}, \mathrm{C}\right), 73.1(\mathrm{CH}), 71.1(\mathrm{CH}), 22.0\left(\mathrm{CH}_{3}\right)$, $21.5\left(\mathrm{CH}_{3}\right)$; IR (solid) 3308, 2985, 2941, 1709, 1619, 1514 $\mathrm{cm}^{-1}$.

Diisopropyl 1-(4-Methoxybenzoyl)hydrazine-1,2-dicarboxylate. Compound prepared according to method A. Purification by column chromatography (10-50\% EtOAc/ petrol) afforded diisopropyl 1-(4-methoxybenzoyl)hydrazine1,2-dicarboxylate as a white solid $(1.03 \mathrm{~g}, 3.05 \mathrm{mmol}, 61 \%) .{ }^{1} \mathrm{H}$ NMR $\left(600 \mathrm{MHz} \mathrm{CDCl}_{3}\right) \delta 7.76-7.62(\mathrm{~m}, 2 \mathrm{H}), 6.98-6.64(\mathrm{~m}$, $3 \mathrm{H}$ ), 5.00 (septet, $J=6.2 \mathrm{~Hz}, 1 \mathrm{H}), 4.92$ (septet, $J=6.2 \mathrm{~Hz}, 1 \mathrm{H}$ ), $3.86(\mathrm{~s}, 3 \mathrm{H}), 1.29$ (d, $J=4.5 \mathrm{~Hz}, 6 \mathrm{H}), 1.13(\mathrm{~d}, J=4.5 \mathrm{~Hz}, 6 \mathrm{H})$; ${ }^{13} \mathrm{C}$ NMR (150 MHz, $\left.\mathrm{CDCl}_{3}\right) \delta 170.8(\mathrm{C}), 163.1$ (C), 155.5 (C), $153.3(\mathrm{C}), 131.2(\mathrm{CH}), 127.0(\mathrm{C}), 113.5(\mathrm{CH}), 72.4$
(CH), $70.7(\mathrm{CH}), 55.6\left(\mathrm{CH}_{3}\right), 22.1\left(\mathrm{CH}_{3}\right), 21.6\left(\mathrm{CH}_{3}\right)$; IR (solid) 3310, 2982, 2938, 1733, 1699, 1604, $1510 \mathrm{~cm}^{-1}$.

Diisopropyl 1-(3-Chlorobenzoyl)hydrazine-1,2-dicarboxylate. Compound prepared according to method A. Purification by column chromatography (10-50\% EtOAc/petrol) afforded diisopropyl 1-(3-chlorobenzoyl)hydrazine-1,2-dicarboxylate as a white solid (1.35 g, $3.95 \mathrm{mmol}, 79 \%) .{ }^{1} \mathrm{H} \mathrm{NMR}(700 \mathrm{MHz}$, $\left.\mathrm{CDCl}_{3}\right) \delta 7.76-7.50(\mathrm{~m}, 2 \mathrm{H}), 7.48(\mathrm{~d}, J=7.9 \mathrm{~Hz}, 1 \mathrm{H}), 7.36(\mathrm{~d}, J$ $=7.9 \mathrm{~Hz}, 1 \mathrm{H}), 6.96-6.75(\mathrm{~m}, \mathrm{NH}, 1 \mathrm{H}), 5.01$ (septet, $J=6.2 \mathrm{~Hz}$, $1 \mathrm{H}), 4.91$ (septet, $J=5.6 \mathrm{~Hz}, 1 \mathrm{H}), 1.29(\mathrm{~d}, J=5.8 \mathrm{~Hz}, 6 \mathrm{H})$, 1.14-1.08 (m, 6H); ${ }^{13} \mathrm{C} \mathrm{NMR}\left(175 \mathrm{MHz}, \mathrm{CDCl}_{3}\right) \delta 170.0(\mathrm{C})$, $155.3(\mathrm{C}), 152.7(\mathrm{C}), 137.0(\mathrm{C}), 134.4(\mathrm{CH}), 131.9(\mathrm{CH})$, $129.6(\mathrm{CH}), 128.2(\mathrm{CH}), 126.3(\mathrm{C}), 73.0(\mathrm{CH}), 71.0(\mathrm{CH})$, $22.0\left(\mathrm{CH}_{3}\right), 21.5\left(\mathrm{CH}_{3}\right)$; IR (solid) 3287, 2981, 2940, 2921, $1710,1560 \mathrm{~cm}^{-1}$.

Diisopropyl 1-(2-Chlorobenzoyl)hydrazine-1,2-dicarboxylate 7. Compound prepared according to method A. Purification by column chromatography (10-50\% EtOAc/ petrol) afforded diisopropyl 1-(2-chlorobenzoyl)hydrazine-1,2dicarboxylate as a white solid $(1.49 \mathrm{~g}, 4.35 \mathrm{mmol}, 87 \%) .{ }^{1} \mathrm{H}$ $\operatorname{NMR}\left(700 \mathrm{MHz}, \mathrm{CDCl}_{3}\right) \delta 7.50-7.42(\mathrm{~m}, 1 \mathrm{H}), 7.41-7.36(\mathrm{~m}$, $2 \mathrm{H}$ ), 7.31 (ddd, $J=7.5,6.8,1.9 \mathrm{~Hz}, 1 \mathrm{H}), 6.82-6.55(\mathrm{~m}, \mathrm{NH}$, $1 \mathrm{H}), 5.01$ (septet, $J=6.1 \mathrm{~Hz}, 1 \mathrm{H}), 4.93-4.89(\mathrm{~m}, 1 \mathrm{H}), 1.29(\mathrm{~d}, J$ $=6.1 \mathrm{~Hz}, 6 \mathrm{H}), 1.12-1.04(\mathrm{~m}, 6 \mathrm{H}) ;{ }^{13} \mathrm{C} \mathrm{NMR}(175 \mathrm{MHz}$, $\left.\mathrm{CDCl}_{3}\right) \delta 167.7$ (C), 155.1 (C), 151.8 (C), 136.3 (C), 131.0 $(\mathrm{CH}), 130.4(\mathrm{C}), 129.5(\mathrm{CH}), 128.1(\mathrm{CH}), 126.8(\mathrm{CH}), 72.9$ $(\mathrm{CH}), 70.9(\mathrm{CH}), 22.0\left(\mathrm{CH}_{3}\right), 21.4\left(\mathrm{CH}_{3}\right), \mathrm{IR}$ (solid) 3298, 2979, 2944, 2855, 1739, 1710, 1613, $1572 \mathrm{~cm}^{-1}$.

Diisopropyl 1-(2,3,4-Trimethoxybenzoyl)hydrazine-1,2-dicarboxylate. Compound prepared according to method A. Purification by column chromatography (10-50\% EtOAc/ petrol) afforded diisopropyl 1-(2,3,4-trimethoxybenzoyl)hydrazine-1,2-dicarboxylate as a white solid (797 mg, 2.00 mmol, 40\%). Mp 110-112 ${ }^{\circ} \mathrm{C}$; ${ }^{1} \mathrm{H}$ NMR $\left(600 \mathrm{MHz}, \mathrm{CDCl}_{3}\right) \delta$ $7.25-7.08(\mathrm{~m}, 1 \mathrm{H}), 6.88(\mathrm{~s}, \mathrm{NH}, 1 \mathrm{H}), 6.69(\mathrm{~d}, J=8.7 \mathrm{~Hz}, 1 \mathrm{H})$, 5.00-4.88 (m, 2H), $3.92(\mathrm{~s}, 3 \mathrm{H}), 3.89(\mathrm{~s}, 3 \mathrm{H}), 3.87(\mathrm{~s}, 3 \mathrm{H})$, $1.26(\mathrm{~d}, J=5.8 \mathrm{~Hz}, 6 \mathrm{H}), 1.20-1.12(\mathrm{~m}, 6 \mathrm{H}) ;{ }^{13} \mathrm{C}$ NMR $(150$ $\left.\mathrm{MHz}, \mathrm{CDCl}_{3}\right) \delta 168.0(\mathrm{C}), 156.4(\mathrm{C}), 155.1$ (C), 152.5 (C), $151.4(\mathrm{C}), 141.7(\mathrm{C}), 124.3(\mathrm{CH}), 122.9(\mathrm{C}), 107.0(\mathrm{CH}), 72.3$ $(\mathrm{CH}), 70.5(\mathrm{CH}), 62.1\left(\mathrm{CH}_{3}\right), 61.1\left(\mathrm{CH}_{3}\right), 56.2\left(\mathrm{CH}_{3}\right), 22.0$ $\left(\mathrm{CH}_{3}\right), 21.6\left(\mathrm{CH}_{3}\right)$; IR (solid) 3310, 2982, 2940, 1737, 1710, $1596 \mathrm{~cm}^{-1}$; LRMS (ESI) $399\left(30,[\mathrm{M}+\mathrm{H}]^{+}\right), 195(100,[\mathrm{M}-$ $\left.\mathrm{C}_{8} \mathrm{H}_{15} \mathrm{~N}_{2} \mathrm{O}_{4}+\mathrm{H}\right]^{+}$); HRMS (ESI) calcd for $\mathrm{C}_{18} \mathrm{H}_{27} \mathrm{~N}_{2} \mathrm{O}_{8}[\mathrm{M}+$ $\mathrm{H}]^{+}$399.1762; observed 399.1760.

Diisopropyl 1-(3,4-Dimethylbenzoyl)hydrazine-1,2-dicarboxylate. Compound prepared according to method A. Purification by column chromatography (10-50\% EtOAc/ petrol) afforded diisopropyl 1-(3,4-dimethylbenzoyl)hydrazine1,2-dicarboxylate as a white solid $(891 \mathrm{mg}, 2.65 \mathrm{mmol}, 53 \%) .{ }^{1} \mathrm{H}$ $\operatorname{NMR}\left(700 \mathrm{MHz}, \mathrm{CDCl}_{3}\right) \delta 7.47-7.32(\mathrm{~m}, 2 \mathrm{H}), 7.14(\mathrm{~d}, J=7.8$ $\mathrm{Hz}, 1 \mathrm{H}), 7.10-7.00(\mathrm{~m}, \mathrm{NH}, 1 \mathrm{H}), 4.99$ (septet, $J=6.3 \mathrm{~Hz}, 1 \mathrm{H}$ ), 4.88 (septet, $J=6.0 \mathrm{~Hz}, 1 \mathrm{H}), 2.28(\mathrm{~s}, 3 \mathrm{H}), 2.26(\mathrm{~s}, 3 \mathrm{H}), 1.30-$ $1.23(\mathrm{~m}, 6 \mathrm{H}), 1.15-1.04(\mathrm{~m}, 6 \mathrm{H}) ;{ }^{13} \mathrm{C}$ NMR $(150 \mathrm{MHz}$, $\left.\mathrm{CDCl}_{3}\right) \delta 171.4(\mathrm{C}), 155.5$ (C), $153.3(\mathrm{C}), 141.5$ (C), 136.6 (C), $132.6(\mathrm{C}), 129.6(\mathrm{CH}), 129.4(\mathrm{CH}), 126.2(\mathrm{CH}), 72.3$ (CH), $70.6(\mathrm{CH}), 22.0\left(\mathrm{CH}_{3}\right), 21.5\left(\mathrm{CH}_{3}\right), 20.1\left(\mathrm{CH}_{3}\right), 19.7$ $\left(\mathrm{CH}_{3}\right)$; IR (thin film) 3278, 3010, 2986, 1713, 1531, $\mathrm{cm}^{-1}$; LRMS (ESI) $337\left(100,[\mathrm{M}+\mathrm{H}]^{+}\right)$; HRMS (ESI) calcd for $\mathrm{C}_{15} \mathrm{H}_{20} \mathrm{~N}_{3} \mathrm{O}_{7}[\mathrm{M}+\mathrm{H}]^{+}$337.3959, observed 337.3961.

Diisopropyl 1-(3-lodobenzoyl)hydrazine-1,2-dicarboxylate. Compound prepared according to method A. Purification by column chromatography (10-50\% EtOAc/petrol) afforded diisopropyl 1-(3-iodobenzoyl)hydrazine-1,2-dicarboxylate as a 
white solid (1.11 g, $2.55 \mathrm{mmol}, 51 \%) .{ }^{1} \mathrm{H}$ NMR (700 MHz, $\left.\mathrm{CDCl}_{3}\right) \delta 8.01-7.90(\mathrm{~m}, 1 \mathrm{H}), 7.84(\mathrm{~d}, J=7.9 \mathrm{~Hz}, 1 \mathrm{H}), 7.71-$ $7.53(\mathrm{~m}, 1 \mathrm{H}), 7.16(\mathrm{t}, J=7.8 \mathrm{~Hz}, 1 \mathrm{H}), 5.01$ (septet, $J=6.2 \mathrm{~Hz}$, $1 \mathrm{H}), 4.92$ (septet, $J=6.1 \mathrm{~Hz}, 1 \mathrm{H}), 1.30(\mathrm{~d}, J=5.9 \mathrm{~Hz}, 6 \mathrm{H})$, $1.13-1.08(\mathrm{~m}, 6 \mathrm{H}) ;{ }^{13} \mathrm{C} \mathrm{NMR}\left(175 \mathrm{MHz}, \mathrm{CDCl}_{3}\right) \delta 169.6(\mathrm{C})$, $155.3(\mathrm{C}), 152.6(\mathrm{C}), 140.8(\mathrm{CH}), 136.8(\mathrm{CH}), 129.9(\mathrm{CH})$, $127.3(\mathrm{CH}), 93.4(\mathrm{CH}), 73.0(\mathrm{CH}), 71.0(\mathrm{CH}), 22.1\left(\mathrm{CH}_{3}\right)$, $21.5\left(\mathrm{CH}_{3}\right)$; IR (solid) 3300, 2975, 2941, $1711,1535 \mathrm{~cm}^{-1}$.

(2-Bromophenoxy)trimethylsilane. To a solution of 2bromophenol $(603 \mu \mathrm{L}, 5.70 \mathrm{mmol})$ in THF $(10 \mathrm{~mL})$ was added hexamethyldisilazane $(1.57 \mathrm{~mL}, 7.50 \mathrm{mmol})$. The solution was refluxed for $2 \mathrm{~h}$ and then allowed to cool down to room temperature. The solvent was then removed in vacuo to afford (2-bromophenoxy)trimethylsilane as an orange oil (1.24 g, $5.10 \mathrm{mmol}, 89 \%) .{ }^{1} \mathrm{H} \mathrm{NMR}\left(600 \mathrm{MHz}, \mathrm{CDCl}_{3}\right) \delta 7.53$ (dd, $J$ $=7.9,1.6 \mathrm{~Hz}, 1 \mathrm{H}), 7.18(\mathrm{td}, J=8.0,1.6 \mathrm{~Hz}, 1 \mathrm{H}), 6.88(\mathrm{dd}, J=$ $8.0,1.5 \mathrm{~Hz}, 1 \mathrm{H}), 6.85(\mathrm{td}, J=7.8,1.5 \mathrm{~Hz}, 1 \mathrm{H}), 0.31(\mathrm{~s}, 9 \mathrm{H}) ;{ }^{13} \mathrm{C}$ NMR $\left(150 \mathrm{MHz}, \mathrm{CDCl}_{3}\right) \delta 152.6(\mathrm{C}), 133.4(\mathrm{CH}), 128.4$ $(\mathrm{CH}), 122.8(\mathrm{CH}), 120.9(\mathrm{CH}), 115.7(\mathrm{C}),-0.5\left(\mathrm{CH}_{3}\right)$; IR (thin film) $3056,2986,1584 \mathrm{~cm}^{-1}$.

2-(Trimethylsilyl)phenyl Trifluoromethanesulfonate. To a solution of (2-bromophenoxy)trimethylsilane (2.00 g, 8.60 $\mathrm{mmol})$ in THF $(20 \mathrm{~mL})$ at $-78^{\circ} \mathrm{C}$ was added dropwise $n$-BuLi $(2.5 \mathrm{M}, 3.91 \mathrm{~mL}, 12.2 \mathrm{mmol})$. The reaction mixture was stirred for $20 \mathrm{~min}$. After this time, to the solution was added dropwise $\mathrm{Tf}_{2} \mathrm{O}(1.90 \mathrm{~mL}, 12.2 \mathrm{mmol})$. The reaction was allowed to warm slowly to room temperature and stirred for a further $30 \mathrm{~min}$. The solution was quenched with $\mathrm{NaHCO}_{3}$ and extracted with EtOAc. The combined organic extracts were dried $\left(\mathrm{MgSO}_{4}\right)$ and filtered, and the solvent removed in vacuo. Purification by column chromatography (10-80\% EtOAc/petrol) afforded 2(trimethylsilyl)phenyl trifluoromethanesulfonate as a yellow oil (1.46 g, $4.90 \mathrm{mmol}, 57 \%) .{ }^{1} \mathrm{H} \mathrm{NMR}\left(600 \mathrm{MHz}, \mathrm{CDCl}_{3}\right) \delta 7.54$ $(\mathrm{d}, J=7.3 \mathrm{~Hz}, 1 \mathrm{H}), 7.45(\mathrm{td}, J=7.8,1.5 \mathrm{~Hz}, 1 \mathrm{H}), 7.36-7.33(\mathrm{~m}$, 2H), $0.37(\mathrm{~s}, 9 \mathrm{H}) ;{ }^{13} \mathrm{C}$ NMR (150 MHz, $\left.\mathrm{CDCl}_{3}\right) \delta 155.2(\mathrm{C})$, $136.4(\mathrm{CH}), 132.7(\mathrm{CH}), 131.4(\mathrm{CH}), 127.6(\mathrm{CH}), 119.6$ $(\mathrm{CH}), 118.6\left(\mathrm{q}, J_{\mathrm{C}-\mathrm{F}}=319.9 \mathrm{~Hz}, \mathrm{C}\right),-0.7\left(\mathrm{CH}_{3}\right)$; IR (thin film) $3054,2987 \mathrm{~cm}^{-1}$.

General Experimental for the Formation of 2Hydrazobenzophenones 1a-j: Method B. To a solution of acyl hydrazide ( $500 \mu \mathrm{mol}, 1.0$ equiv) and TBAT $(1.00 \mathrm{mmol}$, $540 \mathrm{mg}, 2.0$ equiv) in toluene $(6 \mathrm{~mL})$ was added 2 (trimethylsilyl)phenyl trifluoromethanesulfonate $(750 \mu \mathrm{mol}$, $182 \mu \mathrm{L}, 1.5$ equiv). The reaction mixture was stirred at $50^{\circ} \mathrm{C}$ for $16 \mathrm{~h}$. The resulting solution was then allowed to cool down to room temperature, and the solvent was evaporated in vacuo. The resultant crude residue was purified as described below. These 2hydrazobenzophenone compounds exist as rotamers, and this leads to broadness in peaks + additional peaks (the stereodynamics of the $\mathrm{N}-\mathrm{N}$ bond about hydrazide-type molecules is well known in the literature; see for example: ref 40). As such, we conducted VT NMR experiments on a model compound (i.e., compound 1a) to prove that the additional peaks for the 2hydrazobenzophenones at room temperature were indeed very likely to be due to the rotameric nature of these molecules.

Diisopropyl 1-(2-(4-Fluorobenzoyl)phenyl)hydrazine-1,2dicarboxylate 1a. Compound prepared according to method B. Purification by column chromatography (10-50\% EtOAc/ petrol) afforded diisopropyl 1-(2-(4-fluorobenzoyl)phenyl)hydrazine-1,2-dicarboxylate as a pale yellow oil $(173 \mathrm{mg}, 430$ $\mu \mathrm{mol}, 86 \%) .{ }^{1} \mathrm{H}$ NMR $\left(700 \mathrm{MHz}, \mathrm{CDCl}_{3}\right) \delta 7.83-7.81(\mathrm{~m}$, $2 \mathrm{H}), 7.77-7.76(\mathrm{~m}, 1 \mathrm{H}), 7.57-7.54(\mathrm{~m}, 1 \mathrm{H}), 7.42-7.35(\mathrm{~m}$, $2 \mathrm{H}), 7.12(\mathrm{t}, J=7.6 \mathrm{~Hz}, 2 \mathrm{H}), 7.12($ br s, $1 \mathrm{H}, \mathrm{NH}), 5.00-4.93$ $(\mathrm{m}, 1 \mathrm{H}), 4.79$ (septet, $J=6.1 \mathrm{~Hz}, 1 \mathrm{H}), 1.31-1.22(\mathrm{~m}, 6 \mathrm{H})$, 1.16-0.90 (m, 6H); $\left.{ }^{13} \mathrm{C} \mathrm{NMR} \mathrm{(175} \mathrm{MHz,} \mathrm{CDCl}_{3}\right) \delta 195.1(\mathrm{C})$, $194.5(\mathrm{C}), 166.1\left(\mathrm{~d}, J_{\mathrm{C}-\mathrm{F}}=253.8 \mathrm{~Hz}, \mathrm{C}\right), 165.9\left(\mathrm{~d}, J_{\mathrm{C}-\mathrm{F}}=253.7\right.$ $\mathrm{Hz}, \mathrm{C}), 156.1$ (C), 155.8 (C), 155.0 (C), 154.5 (C), 141.0 (C), $135.4(\mathrm{C}), 133.5(\mathrm{C}), 133.4(\mathrm{C}), 133.1(\mathrm{CH}), 132.4(\mathrm{CH})$, $130.1(\mathrm{CH}), 129.8(\mathrm{CH}), 129.4(\mathrm{CH}), 128.9(\mathrm{CH}), 127.7$ $(\mathrm{CH}), 127.4(\mathrm{CH}), 115.7\left(\mathrm{~d}, J_{\mathrm{C}-\mathrm{F}}=21.7 \mathrm{~Hz}\right), 71.2(\mathrm{CH}), 70.8$ $(\mathrm{CH}), 70.0(\mathrm{CH}), 22.1\left(\mathrm{CH}_{3}\right), 22.0\left(\mathrm{CH}_{3}\right), 21.8\left(\mathrm{CH}_{3}\right)$; IR (thin film) 3323, 2921, 2834, 1711, 1666, 1622, 1599, 1574 $\mathrm{cm}^{-1}$; LRMS (ESI) $403\left(100,[\mathrm{M}+\mathrm{H}]^{+}\right)$; HRMS (ESI) calcd for $\mathrm{C}_{21} \mathrm{H}_{24} \mathrm{FN}_{2} \mathrm{O}_{5}[\mathrm{M}+\mathrm{H}]^{+}$403.1664; observed 403.1658.

${ }^{1} \mathrm{H}$ NMR $\left(400 \mathrm{MHz}, \mathrm{CDCl}_{3},-57^{\circ} \mathrm{C}\right) \delta 7.88-7.72(\mathrm{~m}, 3 \mathrm{H})$, $7.62-7.58(\mathrm{~m}, 1 \mathrm{H}), 7.45-7.35(\mathrm{~m}, 3 \mathrm{H}), 7.20-7.13(\mathrm{~m}, 2 \mathrm{H})$, 5.00-4.72 (m, 2H), 1.38-0.75 (m, 12H).

${ }^{1} \mathrm{H}$ NMR (400 MHz, DMSO- $\left.d_{6}, 120{ }^{\circ} \mathrm{C}\right) \delta 8.84($ br s, $1 \mathrm{H})$, $7.78-7.75(\mathrm{~m}, 2 \mathrm{H}), 7.59$ (ddd, $J=8.1,7.4,1.6 \mathrm{~Hz}, 1 \mathrm{H}), 7.54$ (ddd, $J=8.1,1.2,0.5 \mathrm{~Hz}, 1 \mathrm{H}$ ), 7.39 (ddd, $J=7.6,7.4,1.2 \mathrm{~Hz}$, $1 \mathrm{H}), 7.38$ (ddd, $J=7.6,1.6,0.5 \mathrm{~Hz}, 1 \mathrm{H}), 7.29-7.25(\mathrm{~m}, 2 \mathrm{H})$, 4.84 (septet, $J=6.3 \mathrm{~Hz}, 1 \mathrm{H}$ ), 4.79 (septet, $J=6.3 \mathrm{~Hz}, 1 \mathrm{H}$ ), 1.21 $(\mathrm{d}, J=6.3 \mathrm{~Hz}, 6 \mathrm{H}), 1.06(\mathrm{~d}, J=6.3 \mathrm{~Hz}, 6 \mathrm{H})$.

${ }^{13} \mathrm{C}$ NMR (100 MHz, DMSO- $\left.d_{6}, 120{ }^{\circ} \mathrm{C}\right) \delta 193.0(\mathrm{C}), 165.1$ (d, $\left.J_{\mathrm{C}-\mathrm{F}}=252.1 \mathrm{~Hz}, \mathrm{C}\right), 155.7$ (C), 153.9 (C), 140.4 (C), 134.8 (C), $133.8\left(\mathrm{~d}, J_{\mathrm{C}-\mathrm{F}}=2.8 \mathrm{~Hz}, \mathrm{C}\right), 132.4\left(\mathrm{~d}, J_{\mathrm{C}-\mathrm{F}}=9.5 \mathrm{~Hz}, \mathrm{CH}\right)$, $131.1(\mathrm{CH}), 129.0(\mathrm{CH}), 126.5(\mathrm{CH}), 126.5(\mathrm{CH}), 115.3(\mathrm{~d}$, $\left.J_{\mathrm{C}-\mathrm{F}}=22.2 \mathrm{~Hz}, \mathrm{CH}\right), 70.2(\mathrm{CH}), 68.9(\mathrm{CH}), 21.7\left(\mathrm{CH}_{3}\right), 21.4$ $\left(\mathrm{CH}_{3}\right)$.

Diisopropyl 1-(2-Benzoylphenyl)hydrazine-1,2-dicarboxylate 1b. Compound prepared according to method $\mathrm{B}$. Purification by column chromatography (10-50\% EtOAc/ petrol) afforded diisopropyl 1-(2-benzoylphenyl)hydrazine-1,2dicarboxylate as a pale brown oil $(161 \mathrm{mg}, 420 \mu \mathrm{mol}, 84 \%) .{ }^{1} \mathrm{H}$ NMR (700 MHz, $\mathrm{CDCl}_{3}$ ) 7.80-7.49 (m, 5H), 7.46-7.35 (m, $4 \mathrm{H}), 7.10-6.87(\mathrm{~m}, 1 \mathrm{H}, \mathrm{NH}), 5.06-4.92(\mathrm{~m}, 1 \mathrm{H}), 4.92-4.76$ $(\mathrm{m}, 1 \mathrm{H}), 1.32-1.22(\mathrm{~m}, 6 \mathrm{H}), 1.18-0.92(\mathrm{~m}, 6 \mathrm{H}) ;{ }^{13} \mathrm{C} \mathrm{NMR}$ $\left(175 \mathrm{MHz}, \mathrm{CDCl}_{3}\right) \delta 196.7$ (C), 196.1 (C), 156.1 (C), 155.8 (C), 154.9 (C), 154.5 (C), 153.0 (C), 141.0 (C), 137.3 (C), 137.1 (C), $135.6(\mathrm{C}), 133.6(\mathrm{CH}), 133.2(\mathrm{C}), 132.3(\mathrm{CH})$, $132.0(\mathrm{CH}), 130.4(\mathrm{CH}), 130.3(\mathrm{CH}), 129.7(\mathrm{CH}), 129.5$ $(\mathrm{CH}), 128.5(\mathrm{CH}), 128.3(\mathrm{CH}), 127.6(\mathrm{CH}), 127.4(\mathrm{CH})$, $120.8(\mathrm{CH}), 72.6(\mathrm{CH}), 71.2(\mathrm{CH}), 70.8(\mathrm{CH}), 70.0(\mathrm{CH})$, $69.9(\mathrm{CH}), 22.1\left(\mathrm{CH}_{3}\right), 22.1\left(\mathrm{CH}_{3}\right), 22.0\left(\mathrm{CH}_{3}\right), 21.7\left(\mathrm{CH}_{3}\right)$, $21.5\left(\mathrm{CH}_{3}\right)$; IR (thin film) 3301, 2981, 2937, 1883, 1716, 1659, 1598, $1579 \mathrm{~cm}^{-1}$; LRMS (ESI) $385\left(100,[\mathrm{M}+\mathrm{H}]^{+}\right)$; HRMS (ESI) calcd for $\mathrm{C}_{21} \mathrm{H}_{25} \mathrm{~N}_{2} \mathrm{O}_{5}[\mathrm{M}+\mathrm{H}]^{+}$385.1758; observed 385.1755 .

Diisopropyl 1-(2-(3-Bromobenzoyl)phenyl)hydrazine-1,2dicarboxylate 1c. Compound prepared according to method B. Purification by column chromatography (10-50\% EtOAc/ petrol) afforded diisopropyl 1-(2-(3-bromobenzoyl)phenyl)hydrazine-1,2-dicarboxylate as a clear oil $(183 \mathrm{mg}, 395 \mu \mathrm{mol}$, $79 \%) .{ }^{1} \mathrm{H}$ NMR $\left(600 \mathrm{MHz}, \mathrm{CDCl}_{3}\right) \delta 8.02-7.82(\mathrm{~m}, 1 \mathrm{H})$, $7.77-7.67(\mathrm{~m}, 2 \mathrm{H}), 7.62-7.56(\mathrm{~m}, 1 \mathrm{H}), 7.55-7.10(\mathrm{~m}, 4 \mathrm{H})$, 7.08-6.65 (m, NH, $1 \mathrm{H}), 5.06-4.77(\mathrm{~m}, 2 \mathrm{H}), 3.96-3.93(\mathrm{~m}$, $3 \mathrm{H}), 1.31-0.94(\mathrm{~m}, 12 \mathrm{H}) ;{ }^{13} \mathrm{C} \mathrm{NMR}\left(150 \mathrm{MHz}, \mathrm{CDCl}_{3}\right) \delta$ 195.0 (C), 194.6 (C), 156.2 (C), 155.8 (C), 154.9 (C), 154.5 (C), $142.0(\mathrm{C}), 141.1(\mathrm{C}), 139.1(\mathrm{C}), 136.4(\mathrm{CH}), 136.1(\mathrm{CH})$, $134.8(\mathrm{CH}), 133.3(\mathrm{CH}), 132.7(\mathrm{CH}), 130.1(\mathrm{CH}), 128.9$ (CH), $128.7(\mathrm{CH}), 127.7(\mathrm{CH}), 127.4(\mathrm{CH}), 126.1(\mathrm{C}), 122.8$ (C), $71.3(\mathrm{CH}), 71.0(\mathrm{CH}), 70.1(\mathrm{CH}), 22.2\left(\mathrm{CH}_{3}\right), 22.1$ $\left(\mathrm{CH}_{3}\right), 22.0\left(\mathrm{CH}_{3}\right), 21.8\left(\mathrm{CH}_{3}\right)$; IR (thin film) 3312, 2980, 2880, 1715, 1660, 1622, 1595, $1575 \mathrm{~cm}^{-1}$; LRMS (ESI) 487 (30, $\left.\left[\mathrm{M}^{81} \mathrm{Br}+\mathrm{Na}\right]^{+}\right), 485\left(31,\left[\mathrm{M}^{79} \mathrm{Br}+\mathrm{Na}\right]^{+}\right), 465\left(100,\left[\mathrm{M}^{79} \mathrm{Br}+\right.\right.$ 
$\left.\mathrm{H}]^{+}\right), 463\left(98,\left[\mathrm{M}^{79} \mathrm{Br}+\mathrm{H}\right]^{+}\right)$; HRMS (ESI) calcd for $\mathrm{C}_{21} \mathrm{H}_{24} \mathrm{BrN}_{2} \mathrm{O}_{5}\left[\mathrm{M}^{79} \mathrm{Br}+\mathrm{H}\right]^{+}$463.0863; observed 463.0858.

Diisopropyl 1-(2-(4-(Trifluoromethyl)benzoyl)phenyl)hydrazine-1,2-dicarboxylate 1d. Compound prepared according to method B. Purification by column chromatography (10$50 \%$ EtOAc/petrol) afforded diisopropyl 1-(2-(4(trifluoromethyl)benzoyl)phenyl)hydrazine-1,2-dicarboxylate as a clear oil $(161 \mathrm{mg}, 355 \mu \mathrm{mol}, 71 \%) .{ }^{1} \mathrm{H}$ NMR $(600 \mathrm{MHz}$, $\left.\mathrm{CDCl}_{3}\right) \delta 7.94-7.85(\mathrm{~m}, 2 \mathrm{H}), 7.83-7.63(\mathrm{~m}, 3 \mathrm{H}), 7.62-7.57$ (m, 1H), 7.42-7.34 (m, 2H), 7.09-6.84 (m, NH, 1H), 5.03$4.94(\mathrm{~m}, 1 \mathrm{H}), 4.85-4.75(\mathrm{~m}, 1 \mathrm{H}), 1.30-1.23(\mathrm{~m}, 6 \mathrm{H}), 1.18-$ $0.92(\mathrm{~m}, 6 \mathrm{H}) ;{ }^{13} \mathrm{C} \mathrm{NMR}\left(150 \mathrm{MHz}, \mathrm{CDCl}_{3}\right) \delta 195.4(\mathrm{C}), 194.9$ (C), 155.9 (C), 154.9 (C), 141.1 (C), 140.2 (C), 134.7 (C), $132.9(\mathrm{CH}), 130.6(\mathrm{CH}), 130.0(\mathrm{CH}), 129.9(\mathrm{CH}), 129.2$ $(\mathrm{CH}), 128.7(\mathrm{CH}), 127.7(\mathrm{CH}), 127.4(\mathrm{CH}), 125.6(\mathrm{CH})$, $123.7\left(\mathrm{q}, J_{\mathrm{C}-\mathrm{F}}=272.2 \mathrm{~Hz}, \mathrm{C}\right), 119.0(\mathrm{CH}), 71.3(\mathrm{CH}), 71.0$ (CH), 70.1 (CH), $22.1\left(\mathrm{CH}_{3}\right), 21.9\left(\mathrm{CH}_{3}\right)$; IR (thin film) 3323, 2921, 2834, 1711, 1666, 1622, 1599, $1574 \mathrm{~cm}^{-1}$; LRMS (ESI) $453\left(100,[\mathrm{M}+\mathrm{H}]^{+}\right)$; HRMS (ESI) calcd for $\mathrm{C}_{22} \mathrm{H}_{24} \mathrm{~F}_{3} \mathrm{~N}_{2} \mathrm{O}_{5}[\mathrm{M}$ $+\mathrm{H}]^{+}$453.1632; observed 453.1630.

Diisopropyl 1-(2-(4-Methoxybenzoyl)phenyl)hydrazine1,2-dicarboxylate 1e. Compound prepared according to method B. Purification by column chromatography (10-50\% EtOAc/petrol) afforded diisopropyl 1-(2-(4-methoxybenzoyl)phenyl)hydrazine-1,2-dicarboxylate as a pale brown oil $(151 \mathrm{mg}$, $365 \mu \mathrm{mol}, 73 \%) .{ }^{1} \mathrm{H}$ NMR $\left(700 \mathrm{MHz}, \mathrm{CDCl}_{3}\right) \delta 7.77-7.57(\mathrm{~m}$, $3 \mathrm{H}), 7.57(\mathrm{t}, J=7.5 \mathrm{~Hz}, 1 \mathrm{H}), 7.41-7.35(\mathrm{~m}, 2 \mathrm{H}), 7.17-7.04$ $(\mathrm{m}, 1 \mathrm{H}, \mathrm{NH}), 6.92(\mathrm{~d}, J=8.6 \mathrm{~Hz}, 1 \mathrm{H}), 5.02-4.93(\mathrm{~m}, 1 \mathrm{H})$, 4.90-4.75 (m, $1 \mathrm{H}), 3.87-3.82(\mathrm{~m}, 3 \mathrm{H}), 1.31-1.20(\mathrm{~m}, 6 \mathrm{H})$, 1.16-0.90 (m, 6H); ${ }^{13} \mathrm{C} \mathrm{NMR}\left(175 \mathrm{MHz}, \mathrm{CDCl}_{3}\right) \delta 195.4(\mathrm{C})$, 194.7 (C), 164.0 (C), 163.9 (C), 163.1 (C), 156.0 (C), 156.1 (C), 155.7 (C), 155.0 (C), 154.6 (C), 140.8 (C), 136.3 (C), $132.9(\mathrm{C}), 131.9(\mathrm{CH}), 131.1(\mathrm{CH}), 131.1(\mathrm{CH}), 129.8(\mathrm{CH})$, $129.6(\mathrm{CH}), 128.9(\mathrm{CH}), 127.6(\mathrm{CH}), 127.4(\mathrm{CH}), 120.8$ $(\mathrm{CH}), 113.8(\mathrm{CH}), 113.5(\mathrm{CH}), 72.6(\mathrm{CH}), 72.3(\mathrm{CH}), 70.7$ $(\mathrm{CH}), 70.6(\mathrm{CH}), 69.9(\mathrm{CH}), 55.7\left(\mathrm{CH}_{3}\right), 55.6\left(\mathrm{CH}_{3}\right), 55.6$ $\left(\mathrm{CH}_{3}\right), 22.1\left(\mathrm{CH}_{3}\right), 22.1\left(\mathrm{CH}_{3}\right), 22.0\left(\mathrm{CH}_{3}\right), 21.7\left(\mathrm{CH}_{3}\right), 21.6$ $\left(\mathrm{CH}_{3}\right)$; IR (thin film) 3302, 2981, 2937, 2842, 1717, 1652, 1597, $1577 \mathrm{~cm}^{-1}$; LRMS (ESI) $437\left(30,[\mathrm{M}+\mathrm{Na}]^{+}\right), 415(100,[\mathrm{M}+$ $\mathrm{H}]^{+}$); HRMS (ESI) calcd for $\mathrm{C}_{22} \mathrm{H}_{27} \mathrm{~N}_{2} \mathrm{O}_{6}[\mathrm{M}+\mathrm{H}]^{+}$415.1864; observed 415.1862.

Diisopropyl 1-(2-(3-Chlorobenzoyl)phenyl)hydrazine-1,2dicarboxylate 1f. Compound prepared according to method B. Purification by column chromatography (10-50\% EtOAc/ petrol) afforded diisopropyl 1-(2-(3-chlorobenzoyl)phenyl)hydrazine-1,2-dicarboxylate as a pale yellow oil $(170 \mathrm{mg}, 405$ $\mu$ mol, 81\%). ${ }^{1} \mathrm{H}$ NMR (700 MHz, $\left.\mathrm{CDCl}_{3}\right) \delta 7.80-7.64(\mathrm{~m}$, $2 \mathrm{H}), 7.62-7.46(\mathrm{~m}, 2 \mathrm{H}), 7.45-7.34(\mathrm{~m}, 3 \mathrm{H}), 7.34-7.14(\mathrm{~m}$, $1 \mathrm{H}),(\mathrm{m}, 1 \mathrm{H}, \mathrm{NH}), 5.07-4.80(\mathrm{~m}, 2 \mathrm{H}), 1.30-0.94(\mathrm{~m}, 12 \mathrm{H})$; ${ }^{13} \mathrm{C}$ NMR (175 MHz, $\left.\mathrm{CDCl}_{3}\right) \delta 195.1$ (C), 194.7 (C), 156.2 (C), 156.1 (C), 155.9 (C), 154.9 (C), 154.5 (C), 142.0 (C), 141.0 (C), 138.9 (C), 138.7 (C), $134.8(\mathrm{C}), 133.5(\mathrm{CH}), 133.2$ $(\mathrm{CH}), 132.7(\mathrm{CH}), 130.4(\mathrm{CH}), 130.0(\mathrm{CH}), 129.9(\mathrm{CH})$, $129.2(\mathrm{CH}), 128.7(\mathrm{CH}), 128.4(\mathrm{CH}), 127.8(\mathrm{CH}), 127.4$ (CH), $126.2(\mathrm{CH}), 120.8(\mathrm{CH}), 71.3(\mathrm{CH}), 71.0(\mathrm{CH}), 70.1$ (CH), $22.1\left(\mathrm{CH}_{3}\right), 22.1\left(\mathrm{CH}_{3}\right), 22.0\left(\mathrm{CH}_{3}\right), 22.0\left(\mathrm{CH}_{3}\right), 21.8$ $\left(\mathrm{CH}_{3}\right)$; IR (thin film) 3305, 2985, 2884, 1718, 1658, 1619, 1599, $1580 \mathrm{~cm}^{-1}$; LRMS (ESI) $443\left(8,\left[\mathrm{M}^{37} \mathrm{Cl}+\mathrm{Na}\right]^{+}\right), 441(25$, $\left.\left[\mathrm{M}^{35} \mathrm{Cl}+\mathrm{Na}\right]^{+}\right), 421\left(30,\left[\mathrm{M}^{37} \mathrm{Cl}+\mathrm{H}\right]^{+}\right), 419\left(100,\left[\mathrm{M}^{35} \mathrm{Cl}+\right.\right.$ $\mathrm{H}]^{+}$); HRMS (ESI) calcd for $\mathrm{C}_{21} \mathrm{H}_{24} \mathrm{ClN}_{2} \mathrm{O}_{5}\left[\mathrm{M}^{35} \mathrm{Cl}+\mathrm{H}\right]^{+}$ 419.1368; observed 419.1367.

Diisopropyl 1-(2-(2-Chlorobenzoyl)phenyl)hydrazine-1,2dicarboxylate 1g. Compound prepared according to method
B. Purification by column chromatography (10-50\% EtOAc/ petrol) afforded diisopropyl 1-(2-(2-chlorobenzoyl)phenyl)hydrazine-1,2-dicarboxylate as a clear oil $(140 \mathrm{mg}, 335 \mu \mathrm{mol}$, 67\%). ${ }^{1} \mathrm{H}$ NMR (700 MHz, $\left.\mathrm{CDCl}_{3}\right) \delta 7.78-6.70(\mathrm{~m}, 9 \mathrm{H})$, 5.05-4.86 (m, 2H), 1.29-1.04 (m, 12H); ${ }^{13} \mathrm{C} \mathrm{NMR} \mathrm{(175} \mathrm{MHz,}$ $\left.\mathrm{CDCl}_{3}\right) \delta 195.5$ (C), 195.1 (C), 156.0 (C), 155.8 (C), 155.1 (C), 154.6 (C), $151.8(\mathrm{C}), 138.2(\mathrm{C}), 136.3(\mathrm{C}), 135.3(\mathrm{CH})$, $135.1(\mathrm{CH}), 134.7(\mathrm{CH}), 134.1(\mathrm{CH}), 134.1(\mathrm{CH}), 133.9$ $(\mathrm{CH}), 133.8(\mathrm{CH}), 132.2(\mathrm{CH}), 132.1(\mathrm{CH}), 132.0(\mathrm{CH})$, $131.9(\mathrm{CH}), 131.0(\mathrm{CH}), 130.8(\mathrm{CH}), 130.7(\mathrm{CH}), 130.6$ $(\mathrm{CH}), 130.5(\mathrm{CH}), 130.4(\mathrm{CH}), 129.9(\mathrm{CH}), 129.5(\mathrm{CH})$, $128.2(\mathrm{CH}), 128.1(\mathrm{CH}), 120.8(\mathrm{CH}), 127.8(\mathrm{CH}), 126.8$ $(\mathrm{CH}), 72.8(\mathrm{CH}), 71.1(\mathrm{CH}), 70.9(\mathrm{CH}), 70.1(\mathrm{CH}), 69.9$ $(\mathrm{CH}), 69.8(\mathrm{CH}), 22.1\left(\mathrm{CH}_{3}\right), 22.0\left(\mathrm{CH}_{3}\right), 22.0\left(\mathrm{CH}_{3}\right), 21.4$ $\left(\mathrm{CH}_{3}\right)$; IR (thin film) 3308, 2992, 2888, 1723, 1653, 1617, 1606 $\mathrm{cm}^{-1}$; LRMS (ESI) $443\left(9,\left[\mathrm{M}^{37} \mathrm{Cl}+\mathrm{Na}\right]^{+}\right), 441\left(26,\left[\mathrm{M}^{35} \mathrm{Cl}+\right.\right.$ $\left.\mathrm{Na}]^{+}\right), 421\left(31,\left[\mathrm{M}^{37} \mathrm{Cl}+\mathrm{H}\right]^{+}\right), 419\left(100,\left[\mathrm{M}^{35} \mathrm{Cl}+\mathrm{H}\right]^{+}\right)$; HRMS (ESI) calcd for $\mathrm{C}_{21} \mathrm{H}_{24} \mathrm{ClN}_{2} \mathrm{O}_{5}\left[\mathrm{M}^{35} \mathrm{Cl}+\mathrm{H}\right]^{+} 419.1368$; observed 419.1370.

Diisopropyl 1-(2-(2,3,4-Trimethoxybenzoyl)phenyl)hydrazine-1,2-dicarboxylate $1 \mathrm{~h}$. Compound prepared according to method B. Purification by column chromatography (10$50 \% \mathrm{EtOAc} /$ petrol) afforded diisopropyl 1-(2-(2,3,4trimethoxybenzoyl)phenyl)hydrazine-1,2-dicarboxylate as a clear oil (171 mg, $360 \mu \mathrm{mol}, 72 \%) .{ }^{1} \mathrm{H}$ NMR (700 MHz, $\left.\mathrm{CDCl}_{3}\right) \delta 7.70-7.52(\mathrm{~m}, 1 \mathrm{H}), 7.52-7.46(\mathrm{~m}, 1 \mathrm{H}), 7.44-7.27$ $(\mathrm{m}, 3 \mathrm{H}), 7.18-7.10(\mathrm{~m}, 1 \mathrm{H}), 6.65(\mathrm{~d}, J=8.7 \mathrm{~Hz}, 1 \mathrm{H}), 4.94-$ $4.78(\mathrm{~m}, 2 \mathrm{H}), 3.87-3.66(\mathrm{~m}, 9 \mathrm{H}), 1.27-1.03(\mathrm{~m}, 12 \mathrm{H}) ;{ }^{13} \mathrm{C}$ NMR (175 MHz, $\left.\mathrm{CDCl}_{3}\right) \delta 195.2$ (C), 194.9 (C), 157.4 (C), 157.2 (C), 155.9 (C), 155.6 (C), 155.0 (C), 154.6 (C), 153.7 (C), $142.4(\mathrm{C}), 136.9(\mathrm{C}), 132.6(\mathrm{CH}), 132.3(\mathrm{CH}), 130.9$ $(\mathrm{CH}), 130.6(\mathrm{CH}), 130.1(\mathrm{CH}), 129.9(\mathrm{CH}), 127.6(\mathrm{CH})$, $127.0(\mathrm{CH}), 126.7(\mathrm{CH}), 126.2(\mathrm{CH}), 106.8(\mathrm{CH}), 106.7$ $(\mathrm{CH}), 70.9(\mathrm{CH}), 70.5(\mathrm{CH}), 69.7(\mathrm{CH}), 69.6(\mathrm{CH}), 62.1$ $\left(\mathrm{CH}_{3}\right), 61.7\left(\mathrm{CH}_{3}\right), 61.7\left(\mathrm{CH}_{3}\right), 61.1\left(\mathrm{CH}_{3}\right), 61.0\left(\mathrm{CH}_{3}\right), 56.2$ $\left(\mathrm{CH}_{3}\right), 24.0\left(\mathrm{CH}_{3}\right), 22.2\left(\mathrm{CH}_{3}\right), 22.1\left(\mathrm{CH}_{3}\right), 22.0\left(\mathrm{CH}_{3}\right), 21.8$ $\left(\mathrm{CH}_{3}\right)$; IR (thin film) 3300, 2899, 2815, 1720, 1666, 1614, 1598, $1562 \mathrm{~cm}^{-1}$; LRMS (ESI) $475\left(100,[\mathrm{M}+\mathrm{H}]^{+}\right)$; HRMS (ESI) calcd for $\mathrm{C}_{24} \mathrm{H}_{31} \mathrm{~N}_{2} \mathrm{O}_{8}[\mathrm{M}+\mathrm{H}]^{+} 475.2275$ observed 475.2280.

Diisopropyl 1-(2-(3,4-Dimethylbenzoyl)phenyl)hydrazine1,2-dicarboxylate 1i. Compound prepared according to method B. Purification by column chromatography $(10-50 \%$ EtOAc/petrol) afforded diisopropyl 1-(2-(3,4dimethylbenzoyl)phenyl)hydrazine-1,2-dicarboxylate as a yellow oil $(151 \mathrm{mg}, 365 \mu \mathrm{mol}, 73 \%) .{ }^{1} \mathrm{H}$ NMR $\left(600 \mathrm{MHz} \mathrm{CDCl}_{3}\right)$ $\delta 7.76-7.13(\mathrm{~m}, 8 \mathrm{H}), 5.00-4.77(\mathrm{~m}, 2 \mathrm{H}), 2.31-2.26(\mathrm{~m}, 6 \mathrm{H})$, 1.30-0.92 (m, 12H); $\left.{ }^{13} \mathrm{C} \mathrm{NMR} \mathrm{(150} \mathrm{MHz,} \mathrm{CDCl}_{3}\right) \delta 189.9$ (C), 189.4 (C), 149.4 (C), 149.1 (C), 148.2 (C), 147.9 (C), $146.5(\mathrm{C}), 136.5(\mathrm{CH}), 136.2(\mathrm{CH}), 134.8(\mathrm{C}), 134.2(\mathrm{CH})$, $130.2(\mathrm{CH}), 129.3(\mathrm{CH}), 128.3(\mathrm{CH}), 125.8(\mathrm{CH}), 125.3$ $(\mathrm{CH}), 124.6(\mathrm{CH}), 122.9(\mathrm{CH}), 121.8(\mathrm{CH}), 121.6(\mathrm{CH})$, $120.8(\mathrm{CH}), 119.4(\mathrm{CH}), 65.6(\mathrm{CH}), 64.3(\mathrm{CH}), 63.9(\mathrm{CH})$, $63.2(\mathrm{CH}), 15.3\left(\mathrm{CH}_{3}\right), 14.0\left(\mathrm{CH}_{3}\right), 13.4\left(\mathrm{CH}_{3}\right)$; IR (thin film) 3329, 2921, 2840, 1711, 1653, 1622, 1591, $1569 \mathrm{~cm}^{-1}$; LRMS (ESI) $413\left(100,[\mathrm{M}+\mathrm{H}]^{+}\right)$; HRMS (ESI) calcd for $\mathrm{C}_{23} \mathrm{H}_{29} \mathrm{~N}_{2} \mathrm{O}_{5}$ $[\mathrm{M}+\mathrm{H}]^{+}$413.2071; observed 413.2068.

Diisopropyl 1-(2-(3-lodobenzoyl)phenyl)hydrazine-1,2-dicarboxylate $1 \mathrm{j}$. Compound prepared according to method B. Purification by column chromatography (10-50\% EtOAc/ petrol) afforded diisopropyl 1-(2-(3-iodobenzoyl)phenyl)hydrazine-1,2-dicarboxylate as a clear oil $(179 \mathrm{mg}, 350 \mu \mathrm{mol}$, 70\%). ${ }^{1} \mathrm{H}$ NMR $\left(600 \mathrm{MHz}, \mathrm{CDCl}_{3}\right) \delta 8.20-8.04(\mathrm{~m}, 1 \mathrm{H})$, 7.95-7.87 (m, 1H), 7.82-7.67 (m, 2H), 7.60-7.57 (m, 1H), 
7.47-7.30 (m, 3H), 7.22-7.17 (m, 1H), 7.08-6.84 (m, NH, $1 \mathrm{H}), 5.02-4.79(\mathrm{~m}, 2 \mathrm{H}), 1.39-0.96(\mathrm{~m}, 12 \mathrm{H}) ;{ }^{13} \mathrm{C}$ NMR (150 $\left.\mathrm{MHz}, \mathrm{CDCl}_{3}\right) \delta 194.9$ (C), 194.6 (C), 156.2 (C), 156.1 (C), 155.9 (C), 154.9 (C), 154.5 (C), 142.3 (C), 141.9 (C), 141.1 (C), $141.0(\mathrm{C}), 139.2(\mathrm{CH}), 138.7(\mathrm{CH}), 136.7(\mathrm{CH}), 132.7$ $(\mathrm{CH}), 130.2(\mathrm{CH}), 129.8(\mathrm{CH}), 129.5(\mathrm{CH}), 129.2(\mathrm{CH})$, $128.7(\mathrm{CH}), 127.7(\mathrm{CH}), 127.4(\mathrm{CH}), 120.8(\mathrm{CH}), 94.2(\mathrm{C})$, $93.4(\mathrm{C}), 71.3(\mathrm{CH}), 71.0(\mathrm{CH}), 70.1(\mathrm{CH}), 22.1\left(\mathrm{CH}_{3}\right), 21.1$ $\left(\mathrm{CH}_{3}\right), 21.1\left(\mathrm{CH}_{3}\right), 22.0\left(\mathrm{CH}_{3}\right) 21.8\left(\mathrm{CH}_{3}\right), 21.6\left(\mathrm{CH}_{3}\right), 21.5$ $\left(\mathrm{CH}_{3}\right)$; IR (thin film) 3310, 2906, 2844, 1711, 1652, 1619, 1597 $\mathrm{cm}^{-1}$; LRMS (ESI) $511\left(100,[\mathrm{M}+\mathrm{H}]^{+}\right)$; HRMS (ESI) calcd for $\mathrm{C}_{21} \mathrm{H}_{24} \mathrm{IN}_{2} \mathrm{O}_{5}[\mathrm{M}+\mathrm{H}]^{+}$511.0725; observed 511.0720.

General Experimental for the Formation of Isopropyl Carbamate Protected 2-Aminobenzophenones 3a-j: Method C. To a stirring solution of sodium hydride $(60 \%$ mineral oil dispersion, $50 \mathrm{mg}, 1.25 \mathrm{mmol}, 2.5$ equiv) in anhydrous THF $(0.5 \mathrm{~mL})$ under an atmosphere of argon was added dropwise a solution of 2-hydrazobenzophenone ( 0.5 mmol, 1 equiv) predissolved in anhydrous THF $(1 \mathrm{~mL})$, and the reaction mixture was stirred for $5 \mathrm{~min}$. After this time, to the reaction mixture was added diethyl bromomalonate $(205 \mu \mathrm{L}$, $1.25 \mathrm{mmol}, 2.5$ equiv) predissolved in $1.5 \mathrm{~mL}$ anhydrous THF via a syringe pump (over $30 \mathrm{~min}, 5.7 \mu \mathrm{L} \mathrm{min}{ }^{-1}$ ). The reaction mixture was then stirred at $20^{\circ} \mathrm{C}$ for $4 \mathrm{~h}$ and monitored via TLC till completion and poured over sat. aq. $\mathrm{NH}_{4} \mathrm{Cl}(10 \mathrm{~mL})$. The resulting solution was extracted with EtOAc $(3 \times 15 \mathrm{~mL})$. The combined organic extracts were dried $\left(\mathrm{MgSO}_{4}\right)$ and filtered, and the solvent was evaporated in vacuo. The resultant residue was purified as described below.

Isopropyl (2-(4-Fluorobenzoyl)phenyl)carbamate $3 a$. Compound prepared according to method C. Purification by column chromatography $(10-50 \%$ EtOAc/petrol) yielded isopropyl (2-(4-fluorobenzoyl)phenyl)carbamate as a yellow oil (123 mg, $0.410 \mathrm{mmol}, 82 \%) .{ }^{1} \mathrm{H} \mathrm{NMR}\left(500 \mathrm{MHz}, \mathrm{CDCl}_{3}\right) \delta$ $9.99(\mathrm{br} \mathrm{s}, \mathrm{NH}, 1 \mathrm{H}), 8.42(\mathrm{~d}, J=8.4 \mathrm{~Hz}, 1 \mathrm{H}), 7.74(\mathrm{dd}, J=8.8$, $5.4 \mathrm{~Hz}, 2 \mathrm{H}), 7.59-7.53(\mathrm{~m}, 1 \mathrm{H}), 7.49(\mathrm{dd}, J=7.9,1.5 \mathrm{~Hz}, 1 \mathrm{H})$, $7.16(\mathrm{t}, J=8.6 \mathrm{~Hz}, 2 \mathrm{H}), 7.03(\mathrm{t}, J=8.1 \mathrm{~Hz}, 1 \mathrm{H}), 5.01$ (septet, $J=$ $6.3 \mathrm{~Hz}, 1 \mathrm{H}), 1.30(\mathrm{~d}, J=6.3 \mathrm{~Hz}, 6 \mathrm{H}) ;{ }^{13} \mathrm{C} \mathrm{NMR}(125 \mathrm{MHz}$, $\left.\mathrm{CDCl}_{3}\right) \delta 197.7(\mathrm{C}), 165.4\left(\mathrm{~d}, J_{\mathrm{C}-\mathrm{F}}=254.4 \mathrm{~Hz}, \mathrm{C}\right), 153.5(\mathrm{C})$, $141.1(\mathrm{C}), 135.0(\mathrm{C}), 134.3(\mathrm{CH}), 133.2(\mathrm{CH}), 132.6\left(\mathrm{~d}, J_{\mathrm{C}-\mathrm{F}}=\right.$ $9.2 \mathrm{~Hz}, \mathrm{CH}), 123.0(\mathrm{CH}), 121.1(\mathrm{CH}), 120.2(\mathrm{C}), 115.5(\mathrm{~d}$, $\left.J_{\mathrm{C}-\mathrm{F}}=21.8 \mathrm{~Hz}, \mathrm{CH}\right), 68.9(\mathrm{CH}), 22.1\left(\mathrm{CH}_{3}\right)$; IR (thin film) 3302, 2981, 2935, 1729, 1633, 1580, $1519 \mathrm{~cm}^{-1}$; LRMS (ESI) $302\left(100,[\mathrm{M}+\mathrm{H}]^{+}\right)$; HRMS (ESI) calcd for $\mathrm{C}_{17} \mathrm{H}_{16} \mathrm{FNO}_{3}[\mathrm{M}+$ $\mathrm{H}]^{+}$302.1192; observed 302.1193.

Isopropyl (2-Benzoylphenyl)carbamate 3b. Compound prepared according to method C. Purification by column chromatography (10-50\% EtOAc/petrol) yielded isopropyl (2benzoylphenyl)carbamate as a colorless oil $(110 \mathrm{mg}, 0.390$ mmol, 78\%). ${ }^{1} \mathrm{H}$ NMR (500 MHz, $\left.\mathrm{CDCl}_{3}\right) \delta 10.17$ (br s, NH, $1 \mathrm{H}), 8.44(\mathrm{~d}, J=8.7 \mathrm{~Hz}, 1 \mathrm{H}), 7.70(\mathrm{dt}, J=8.4,1.5 \mathrm{~Hz}, 2 \mathrm{H})$, $7.61-7.43(\mathrm{~m}, 5 \mathrm{H}), 7.04-6.98(\mathrm{~m}, 1 \mathrm{H}), 5.02$ (septet, $J=6.3 \mathrm{~Hz}$, $1 \mathrm{H}), 1.30(\mathrm{~d}, J=6.3 \mathrm{~Hz}, 6 \mathrm{H}) ;{ }^{13} \mathrm{C} \mathrm{NMR}\left(125 \mathrm{MHz}, \mathrm{CDCl}_{3}\right) \delta$ 199.4 (C), 153.6 (C), 141.3 (C), 138.9 (C), $134.3(\mathrm{CH}), 133.7$ $(\mathrm{CH}), 132.5(\mathrm{CH}), 130.0(\mathrm{CH}), 128.4(\mathrm{CH}), 122.9(\mathrm{C}), 121.0$ $(\mathrm{CH}), 120.0(\mathrm{CH}), 68.9(\mathrm{CH}), 22.2\left(\mathrm{CH}_{3}\right)$; IR (thin film) 3318, 2979, 2934, 1728, 1635, 1580, $1518 \mathrm{~cm}^{-1}$; LRMS (ESI) $284\left(100,[\mathrm{M}+\mathrm{H}]^{+}\right)$; HRMS (ESI) calcd for $\mathrm{C}_{17} \mathrm{H}_{18} \mathrm{NO}_{3}[\mathrm{M}+$ $\mathrm{H}]^{+}$284.1287; observed 284.1286.

Isopropyl (2-(3-Bromobenzoyl)phenyl)carbamate 3c. Compound prepared according to method C. Purification by column chromatography $(10-50 \%$ EtOAc/petrol) yielded isopropyl (2-(3-bromobenzoyl)phenyl)carbamate as a brown oil (123 mg, $0.340 \mathrm{mmol}, 68 \%) .{ }^{1} \mathrm{H} \mathrm{NMR}\left(700 \mathrm{MHz}, \mathrm{CDCl}_{3}\right) \delta$ 10.11 (br s, NH, $1 \mathrm{H}), 8.44(\mathrm{~d}, J=7.9 \mathrm{~Hz}, 1 \mathrm{H}), 7.83(\mathrm{t}, J=1.7$ $\mathrm{Hz}, 1 \mathrm{H}), 7.70(\mathrm{ddd}, J=8.0,2.0,1.0 \mathrm{~Hz}, 1 \mathrm{H}), 7.60-7.58(\mathrm{~m}$, $1 \mathrm{H}), 7.56(\mathrm{dd}, J=8.6,7.5,1.5 \mathrm{~Hz}, 1 \mathrm{H}), 7.49(\mathrm{dd}, J=7.9,1.5 \mathrm{~Hz}$, $1 \mathrm{H}), 7.35(\mathrm{t}, J=7.9 \mathrm{~Hz}, 1 \mathrm{H}), 7.03(\mathrm{~m}, 1 \mathrm{H}), 5.02$ (septet, $J=6.3$ $\mathrm{Hz}, 1 \mathrm{H}), 1.30(\mathrm{~d}, J=6.3 \mathrm{~Hz}, 6 \mathrm{H}) ;{ }^{13} \mathrm{C} \mathrm{NMR}\left(175 \mathrm{MHz}, \mathrm{CDCl}_{3}\right)$ $\delta 197.8(\mathrm{C}), 153.5(\mathrm{C}), 141.5(\mathrm{C}), 140.8(\mathrm{C}), 135.3(\mathrm{CH})$, $134.8(\mathrm{CH}), 133.5(\mathrm{CH}), 132.7(\mathrm{CH}), 129.9(\mathrm{CH}), 128.5$ (CH), 122.7 (C), $122.3(\mathrm{C}), 121.2(\mathrm{CH}), 120.1(\mathrm{CH}), 69.1$ (CH), $22.2\left(\mathrm{CH}_{3}\right)$; IR (thin film) 3310, 3082, 2977, 2934, 1723, 1633, 1603, 1577, 1560, $1510 \mathrm{~cm}^{-1}$; LRMS (ESI) 364 (100, $\left.\left[\mathrm{M}^{81} \mathrm{Br}+\mathrm{H}\right]^{+}\right), 362\left(98,\left[\mathrm{M}^{79} \mathrm{Br}+\mathrm{H}\right]^{+}\right)$; HRMS (ESI) calcd for $\mathrm{C}_{17} \mathrm{H}_{16} \mathrm{BrNO}_{3}\left[\mathrm{M}^{79} \mathrm{Br}+\mathrm{H}\right]^{+}$362.0392; observed 362.0374.

Isopropyl (2-(4-(Trifluoromethyl)benzoyl)phenyl)carbamate $3 d$. Compound prepared according to method C. Purification by column chromatography (10-50\% EtOAc/ petrol) yielded isopropyl (2-(4-(trifluoromethyl)benzoyl)phenyl)carbamate as a yellow oil (125 mg, $0.355 \mathrm{mmol}, 71 \%)$. ${ }^{1} \mathrm{H}$ NMR $\left(700 \mathrm{MHz}, \mathrm{CDCl}_{3}\right) \delta 10.25(\mathrm{br} \mathrm{s}, \mathrm{NH}, 1 \mathrm{H}), 8.47$ (d, $=8.5 \mathrm{~Hz}, 1 \mathrm{H}), 7.77(\mathrm{~d}, J=8.2 \mathrm{~Hz}, 2 \mathrm{H}), 7.73(\mathrm{~d}, J=8.2 \mathrm{~Hz}, 2 \mathrm{H})$, 7.57-7.55 (m, 1H), 7.45 (dd, $J=7.9,1.5 \mathrm{~Hz}, 1 \mathrm{H}), 7.01-6.99$ (m, 1H), 5.02 (septet, $J=6.3 \mathrm{~Hz}, 1 \mathrm{H}), 1.30(\mathrm{~d}, J=6.4 \mathrm{~Hz}, 6 \mathrm{H})$; ${ }^{13} \mathrm{C}$ NMR (175 MHz, $\left.\mathrm{CDCl}_{3}\right) \delta 198.3(\mathrm{C}), 153.3$ (C), 142.4 (C), $141.8(\mathrm{CH}) 135.0(\mathrm{CH}), 133.7(\mathrm{CH}), 133.6\left(\mathrm{q}, J_{\mathrm{C}-\mathrm{F}}=31.0\right.$ $\mathrm{Hz}, \mathrm{C}), 125.4\left(\mathrm{q}, J_{\mathrm{C}-\mathrm{F}}=3.7 \mathrm{~Hz}, \mathrm{CH}\right), 123.8\left(\mathrm{q}, J_{\mathrm{C}-\mathrm{F}}=272.6 \mathrm{~Hz}\right.$, C), $121.9(\mathrm{CH}), 121.2(\mathrm{CH}), 120.1(\mathrm{CH}), 69.1(\mathrm{CH}), 22.2$ $\left(\mathrm{CH}_{3}\right)$; IR (thin film) 3340, 2969, 2920, 1727, 1628, 1590, 1579 $\mathrm{cm}^{-1}$; LRMS (ESI) $352\left(100,[\mathrm{M}+\mathrm{H}]^{+}\right)$; HRMS (ESI) calcd for $\mathrm{C}_{18} \mathrm{H}_{17} \mathrm{~F}_{3} \mathrm{NO}_{3}[\mathrm{M}+\mathrm{H}]^{+} 352.1155$; observed 352.1159.

Isopropyl (2-(4-Methoxybenzoyl)phenyl)carbamate $3 e$. Compound prepared according to method C. Purification by column chromatography $(10-50 \% \mathrm{EtOAc} /$ petrol) yielded isopropyl (2-(4-methoxybenzoyl)phenyl)carbamate as a red oil (119 mg, $0.380 \mathrm{mmol}, 76 \%) .{ }^{1} \mathrm{H}$ NMR (500 MHz, $\left.\mathrm{CDCl}_{3}\right) \delta$ 9.85 (br s, NH, $1 \mathrm{H}), 8.38(\mathrm{~d}, J=8.2 \mathrm{~Hz}, 1 \mathrm{H}), 7.76-7.68(\mathrm{~m}$, $2 \mathrm{H}), 7.56-7.47(\mathrm{~m}, 2 \mathrm{H}), 7.02(\mathrm{t}, J=7.6 \mathrm{~Hz}, 1 \mathrm{H}), 6.98-6.91$ $(\mathrm{m}, 2 \mathrm{H}), 5.00$ (septet, $J=6.3 \mathrm{~Hz}, 1 \mathrm{H}), 3.88(\mathrm{~s}, 3 \mathrm{H}), 1.28(\mathrm{~d}, J=$ $6.3 \mathrm{~Hz}, 1 \mathrm{H}) ;{ }^{13} \mathrm{C}$ NMR $\left(125 \mathrm{MHz}, \mathrm{CDCl}_{3}\right) \delta 197.7$ (C), 163.4 (C), $153.6(\mathrm{C}), 140.6(\mathrm{C}), 133.6(\mathrm{CH}), 132.9(\mathrm{CH}), 132.7$ (CH), $131.2(\mathrm{C}), 123.9(\mathrm{C}), 121.1(\mathrm{CH}), 120.2(\mathrm{CH}), 113.7$ $(\mathrm{CH}), 68.8(\mathrm{CH}), 55.6\left(\mathrm{CH}_{3}\right), 22.2\left(\mathrm{CH}_{3}\right)$; IR (thin film) 3317, 2980, 2935, 2839, 1728, 1628, 1595, $1580 \mathrm{~cm}^{-1}$; LRMS (ESI) $314\left(100,[\mathrm{M}+\mathrm{H}]^{+}\right)$; HRMS (ESI) calcd for $\mathrm{C}_{18} \mathrm{H}_{19} \mathrm{NO}_{4}[\mathrm{M}+$ $\mathrm{H}]^{+}$314.1392; observed 314.1385.

Isopropyl (2-(3-Chlorobenzoyl)phenyl)carbamate $3 f$. Compound prepared according to method C. Purification by column chromatography $(10-50 \% \mathrm{EtOAc} /$ petrol $)$ yielded isopropyl (2-(3-chlorobenzoyl)phenyl)carbamate as a yellow oil (102 mg, $0.320 \mathrm{mmol}, 64 \%) .{ }^{1} \mathrm{H} \mathrm{NMR}\left(700 \mathrm{MHz}, \mathrm{CDCl}_{3}\right) \delta$ 10.12 (br s, NH, 1H), 8.45 (dd, $J=8.5,0.7 \mathrm{~Hz}, 1 \mathrm{H}), 7.68(\mathrm{t}, J=$ $1.7 \mathrm{~Hz}, 1 \mathrm{H}), 7.61-7.53(\mathrm{~m}, 3 \mathrm{H}), 7.49(\mathrm{dd}, J=7.9,1.5 \mathrm{~Hz}, 1 \mathrm{H})$, 7.44-7.38 (m, 1H), 7.06-6.99 (m, 1H), 5.02 (septet, $J=6.3 \mathrm{~Hz}$, $1 \mathrm{H}), 1.30(\mathrm{~d}, J=6.3 \mathrm{~Hz}, 6 \mathrm{H}) ;{ }^{13} \mathrm{C} \mathrm{NMR}\left(175 \mathrm{MHz}, \mathrm{CDCl}_{3}\right) \delta$ 197.9 (C), 153.6 (C), 141.5 (C), 140.6 (C), $134.8(\mathrm{CH}), 134.7$ (C), $133.5(\mathrm{CH}), 132.3(\mathrm{CH}), 129.8(\mathrm{CH}), 129.7(\mathrm{CH}), 128.0$ (CH), $122.3(\mathrm{C}), 121.2(\mathrm{CH}), 120.1(\mathrm{CH}), 69.1(\mathrm{CH}), 22.2$ $\left(\mathrm{CH}_{3}\right)$; IR (thin film) 3313, 3022, 2982, 2936, 1729, 1637, 1604, $1580 \mathrm{~cm}^{-1}$; LRMS (ESI) $320\left(40,\left[\mathrm{M}^{37} \mathrm{Cl}+\mathrm{H}\right]^{+}\right), 318(100$, $\left[\mathrm{M}^{35} \mathrm{Cl}+\mathrm{H}\right]^{+}$); HRMS (ESI) calcd for $\mathrm{C}_{17} \mathrm{H}_{16} \mathrm{ClNO}_{3}\left[\mathrm{M}^{35} \mathrm{Cl}+\right.$ $\mathrm{H}]^{+}$318.0897; observed 318.0893.

Isopropyl (2-(2-Chlorobenzoyl)phenyl)carbamate $3 g$. Compound prepared according to method C. Purification by column chromatography $(10-50 \%$ EtOAc/petrol) yielded 
isopropyl (2-(2-chlorobenzoyl)phenyl)carbamate as a yellow oil (114 mg, $0.36 \mathrm{mmol}, 71 \%) .{ }^{1} \mathrm{H}$ NMR (700 MHz, $\left.\mathrm{CDCl}_{3}\right) \delta$ 10.94 (br s, NH, $1 \mathrm{H}), 8.57(\mathrm{~d}, J=8.5 \mathrm{~Hz}, 1 \mathrm{H}), 7.59-7.54(\mathrm{~m}$, $1 \mathrm{H}), 7.48-7.45(\mathrm{~m}, 1 \mathrm{H}), 7.44(\mathrm{td}, J=7.8,1.6 \mathrm{~Hz}, 1 \mathrm{H}), 7.37(\mathrm{td}$, $J=7.4,1.0 \mathrm{~Hz}, 1 \mathrm{H}), 7.32(\mathrm{ddd}, J=13.7,7.8,1.5 \mathrm{~Hz}, 2 \mathrm{H}), 6.94(\mathrm{t}$, $J=7.6 \mathrm{~Hz}, 1 \mathrm{H}), 5.05$ (septet, $J=6.3 \mathrm{~Hz}, 1 \mathrm{H}), 1.33(\mathrm{~d}, J=6.3 \mathrm{~Hz}$, 6H); ${ }^{13} \mathrm{C}$ NMR (125 MHz, $\left.\mathrm{CDCl}_{3}\right) \delta 198.8(\mathrm{C}), 153.6(\mathrm{C})$, $142.8(\mathrm{C}), 139.1(\mathrm{C}), 135.9(\mathrm{CH}), 134.8(\mathrm{CH}), 131.1(\mathrm{CH})$, $131.0(\mathrm{C}), 130.2(\mathrm{CH}), 128.8(\mathrm{CH}), 126.8(\mathrm{CH}), 121.2(\mathrm{CH})$, $121.0(\mathrm{C}), 119.2(\mathrm{CH}), 69.1(\mathrm{CH}), 22.2\left(\mathrm{CH}_{3}\right)$; IR (thin film) 3320, 3028, 2981, 2936, 1743, 1721, 1635, 1603, $1582 \mathrm{~cm}^{-1}$; LRMS (ESI) $320\left(30,\left[\mathrm{M}^{37} \mathrm{Cl}+\mathrm{H}\right]^{+}\right), 318\left(100,\left[\mathrm{M}^{35} \mathrm{Cl}+\mathrm{H}\right]^{+}\right)$; HRMS (ESI) calcd for $\mathrm{C}_{17} \mathrm{H}_{16} \mathrm{ClNO}_{3}\left[\mathrm{M}^{35} \mathrm{Cl}+\mathrm{H}\right]^{+} 318.0897$; observed 318.0894 .

Isopropyl (2-(2,3,4-Trimethoxybenzoyl)phenyl)carbamate $3 h$. Compound prepared according to method C. Purification by column chromatography (10-50\% EtOAc/petrol) yielded isopropyl (2-(2,3,4-trimethoxybenzoyl)phenyl)carbamate as a yellow oil (134 mg, $0.36 \mathrm{mmol}, 72 \%) .{ }^{1} \mathrm{H}$ NMR (700 MHz, $\left.\mathrm{CDCl}_{3}\right) \delta 10.74($ br s, NH, $1 \mathrm{H}), 8.46(\mathrm{~d}, J=7.9 \mathrm{~Hz}, 1 \mathrm{H}), 7.51-$ $7.47(\mathrm{~m}, 1 \mathrm{H}), 7.43(\mathrm{dd}, J=8.0,1.5 \mathrm{~Hz}, 1 \mathrm{H}), 6.98(\mathrm{~d}, J=8.6 \mathrm{~Hz}$, $1 \mathrm{H}), 6.92-6.90(\mathrm{td}, J=7.4,1.0 \mathrm{~Hz}, 1 \mathrm{H}), 6.69(\mathrm{~d}, J=8.6 \mathrm{~Hz}$, $1 \mathrm{H}), 5.00$ (septet, $J=6.3 \mathrm{~Hz}, 1 \mathrm{H}), 3.89(\mathrm{~s}, 3 \mathrm{H}), 3.86(\mathrm{~s}, 3 \mathrm{H})$, $3.76(\mathrm{~s}, 3 \mathrm{H}), 1.29$ (d, $J=6.3 \mathrm{~Hz}, 6 \mathrm{H}) ;{ }^{13} \mathrm{C} \mathrm{NMR}(175 \mathrm{MHz}$, $\mathrm{CDCl}_{3}$ ) $\delta 198.8$ (C), 156.1 (C), 153.7 (C), 152.3 (C), 142.1 (C), $141.7(\mathrm{C}), 134.8(\mathrm{CH}), 134.3(\mathrm{CH}), 127.1(\mathrm{C}), 124.6$ (CH), $123.0(\mathrm{C}), 120.9(\mathrm{CH}), 119.1(\mathrm{CH}), 106.8(\mathrm{CH}), 68.8$ $(\mathrm{CH}), 61.9(\mathrm{CH}), 61.0(\mathrm{CH}), 56.2(\mathrm{CH}), 22.2\left(\mathrm{CH}_{3}\right)$; IR (thin film) 3301, 3012, 2977, 2929, 1740, 1639, 1593, $1589 \mathrm{~cm}^{-1}$; LRMS (ESI) $374\left(100,[\mathrm{M}+\mathrm{H}]^{+}\right)$; HRMS (ESI) calcd for $\mathrm{C}_{20} \mathrm{H}_{23} \mathrm{NO}_{6}[\mathrm{M}+\mathrm{H}]^{+}$374.1598; observed 374.1601.

Isopropyl (2-(3,4-Dimethylbenzoyl)phenyl)carbamate $3 i$. Compound prepared according to method C. Purification by column chromatography $(10-50 \% \mathrm{EtOAc} /$ petrol $)$ yielded isopropyl (2-(3,4-dimethylbenzoyl)phenyl)carbamate as a brown oil (103 mg, $0.330 \mathrm{mmol}, 66 \%) .{ }^{1} \mathrm{H}$ NMR (500 MHz, $\left.\mathrm{CDCl}_{3}\right) \delta 10.04($ br s, NH, $1 \mathrm{H}), 8.40(\mathrm{~m}, 1 \mathrm{H}), 7.53(\mathrm{~m}, 3 \mathrm{H})$, $7.43(\mathrm{dd}, J=7.8,1.7 \mathrm{~Hz}, 1 \mathrm{H}), 7.22(\mathrm{~d}, J=7.8 \mathrm{~Hz}, 1 \mathrm{H}), 7.06-$ $6.99(\mathrm{~m}, 1 \mathrm{H}), 5.00$ (septet, $J=6.3 \mathrm{~Hz}, 1 \mathrm{H}), 2.35(\mathrm{~s}, 3 \mathrm{H}), 2.32(\mathrm{~s}$, $3 \mathrm{H}), 1.29(\mathrm{~d}, J=6.3 \mathrm{~Hz}, 6 \mathrm{H}) ;{ }^{13} \mathrm{C} \mathrm{NMR}\left(125 \mathrm{MHz}, \mathrm{CDCl}_{3}\right) \delta$ 199.2 (C), 153.6 (C), 142.1 (C), 140.9 (C), 136.8 (C), 136.5 (C), $133.8(\mathrm{CH}), 133.4(\mathrm{CH}), 131.2(\mathrm{CH}), 129.5(\mathrm{CH}), 128.0$ (CH), $123.5(\mathrm{C}), 120.9(\mathrm{CH}), 120.0(\mathrm{CH}), 68.8(\mathrm{CH}), 22.1$ $\left(\mathrm{CH}_{3}\right), 20.1\left(\mathrm{CH}_{3}\right), 19.8\left(\mathrm{CH}_{3}\right)$; IR (thin film) 3286, 2978, 2923, 2853, 1730, 1633, 1600, 1580, $1518 \mathrm{~cm}^{-1}$; LRMS (ESI) $312\left(100,[\mathrm{M}+\mathrm{H}]^{+}\right)$; HRMS (ESI) calcd for $\mathrm{C}_{19} \mathrm{H}_{21} \mathrm{NO}_{3}[\mathrm{M}+$ $\mathrm{H}]^{+}$312.1594; observed 312.1596.

Isopropyl (2-(3-lodobenzoyl)phenyl)carbamate 3j. Compound prepared according to method C. Purification by column chromatography (10-50\% EtOAc/petrol) yielded isopropyl (2(2-chlorobenzoyl)phenyl)carbamate as a yellow oil (139 mg, $0.34 \mathrm{mmol}, 68 \%) .{ }^{1} \mathrm{H}$ NMR (700 MHz, $\left.\mathrm{CDCl}_{3}\right) \delta 10.11$ (br s, $\mathrm{NH}, 1 \mathrm{H}), 8.43(\mathrm{~d}, J=8.5 \mathrm{~Hz}, 1 \mathrm{H}), 8.01(\mathrm{t}, J=1.6 \mathrm{~Hz}, 1 \mathrm{H}), 7.88$ (ddd, $J=7.9,1.7,1.1 \mathrm{~Hz}, 1 \mathrm{H}), 7.61-7.60(\mathrm{~m}, 1 \mathrm{H}), 7.55$ (ddd $J$ $=8.6,7.5,1.4 \mathrm{~Hz}, 1 \mathrm{H}), 7.47(\mathrm{dd}, J=7.9,1.5 \mathrm{~Hz}, 1 \mathrm{H}), 7.19(\mathrm{t}, J=$ $7.8 \mathrm{~Hz}, 2 \mathrm{H}), 7.02-7.00(\mathrm{~m}, 1 \mathrm{H}), 5.01$ (septet, $J=6.3 \mathrm{~Hz}, 1 \mathrm{H}$ ), $1.29(\mathrm{~d}, J=6.3 \mathrm{~Hz}, 6 \mathrm{H}) ;{ }^{13} \mathrm{C} \mathrm{NMR}\left(175 \mathrm{MHz}, \mathrm{CDCl}_{3}\right) \delta 197.6$ (C), $153.5(\mathrm{C}), 141.5(\mathrm{C}), 141.1(\mathrm{C}), 140.8(\mathrm{CH}), 138.5(\mathrm{CH})$, $134.8(\mathrm{CH}), 133.5(\mathrm{C}), 130.0(\mathrm{CH}), 129.0(\mathrm{CH}), 122.2(\mathrm{CH})$, $121.2(\mathrm{CH}), 120.1(\mathrm{C}), 94.1(\mathrm{C}), 69.0(\mathrm{CH}), 22.2\left(\mathrm{CH}_{3}\right)$; IR (thin film) 3318, 3025, 2984, 2940, 1737, 1719, 1640, 1603 $\mathrm{cm}^{-1}$; LRMS (ESI) $410\left(100,[\mathrm{M}+\mathrm{H}]^{+}\right)$; HRMS (ESI) calcd for $\mathrm{C}_{17} \mathrm{H}_{17} \mathrm{INO}_{3}[\mathrm{M}+\mathrm{H}]^{+} 410.0248$; observed 410.0251 .
General Experimental for the Deprotection of Isopropyl Carbamate Protected 2-Aminobenzophenones 5: Method D. To a stirring solution of aluminum chloride (4 mmol, 4 equiv) in dichloromethane $(2 \mathrm{~mL})$ was added dropwise isopropyl carbamate protected 2 -aminobenzophenone $(1 \mathrm{mmol}$, 1 equiv) predissolved in dichloromethane $(0.5 \mathrm{~mL})$. The mixture was then stirred for $1 \mathrm{~h}$ and monitored via TLC till completion. The reaction mixture was then poured into ice water $(10 \mathrm{~mL})$. If a solid precipitate was formed, the mixture was filtered and the solid was washed with water $(3 \times 3 \mathrm{~mL})$ and dried in vacuo. If no solid was formed, the mixture was then extracted with EtOAc $(3 \times 4 \mathrm{~mL})$. The combined organic extracts were then washed with water $(3 \times 4 \mathrm{~mL})$, dried $\left(\mathrm{MgSO}_{4}\right)$, and concentrated in vacuo. The resultant residue was purified as described below.

(2-Aminophenyl)(phenyl)methanone 5b. Compound prepared according to method D. Purification by column chromotography (10-25\% EtOAc/petrol) yielded (2aminophenyl)(phenyl)methanone as a yellow crystalline solid (180 mg, $0.920 \mathrm{mmol}, 92 \%) . \mathrm{Mp} 103-104{ }^{\circ} \mathrm{C} ;{ }^{1} \mathrm{H}$ NMR (700 $\left.\mathrm{MHz}, \mathrm{CDCl}_{3}\right) \delta 7.64(\mathrm{~d}, J=7.8 \mathrm{~Hz}, 2 \mathrm{H}), 7.52(\mathrm{t}, J=7.4 \mathrm{~Hz}, 1 \mathrm{H})$, $7.46(\mathrm{t}, J=7.1 \mathrm{~Hz}, 2 \mathrm{H}), 7.46(\mathrm{t}, J=7.1 \mathrm{~Hz}, 1 \mathrm{H}), 7.29(\mathrm{t}, J=7.7$ $\mathrm{Hz}, 1 \mathrm{H}), 6.73(\mathrm{~d}, J=8.3 \mathrm{~Hz}, 1 \mathrm{H}), 6.60(\mathrm{t}, J=7.5 \mathrm{~Hz}, 1 \mathrm{H}), 6.10$ (br s, NH, 2H); ${ }^{13} \mathrm{C}$ NMR (175 MHz, $\left.\mathrm{CDCl}_{3}\right) \delta 199.2$ (C), $151.1(\mathrm{C}), 140.3(\mathrm{C}), 134.7(\mathrm{CH}), 134.4(\mathrm{CH}), 131.2(\mathrm{CH})$, $129.3(\mathrm{CH}), 128.2(\mathrm{CH}), 118.3(\mathrm{C}), 117.2(\mathrm{CH}), 115.7(\mathrm{CH})$; IR (solid) 3502, 3087, 3056, 3031, 1669, 1593, $1575 \mathrm{~cm}^{-1}$; LRMS (ESI) $198\left(100,[\mathrm{M}+\mathrm{H}]^{+}\right)$; HRMS (ESI) calcd for $\mathrm{C}_{13} \mathrm{H}_{11} \mathrm{NO}[\mathrm{M}+\mathrm{H}]^{+}$198.0919; observed 198.0916.

(2-Aminophenyl)(4-(trifluoromethyl)phenyl)methanone $5 d$. Compound prepared according to method D. Purification by column chromotography (10-25\% EtOAc/petrol) yielded (2-aminophenyl)(4-(trifluoromethyl)phenyl)methanone as a yellow crystalline solid (236 mg, $0.890 \mathrm{mmol}, 89 \%)$. Mp 98$99{ }^{\circ} \mathrm{C}$; ${ }^{1} \mathrm{H} \mathrm{NMR}\left(700 \mathrm{MHz}, \mathrm{CDCl}_{3}\right) \delta 7.74-7.70(\mathrm{~m}, 4 \mathrm{H}), 7.35$ (dd, $J=8.1,1.3 \mathrm{~Hz}, 1 \mathrm{H}), 7.32$ (ddd, $J=8.5,7.0,1.6 \mathrm{~Hz}, 1 \mathrm{H})$, $6.75(\mathrm{dd}, J=8.4,1.0 \mathrm{~Hz}, 1 \mathrm{H}), 6.60(\mathrm{ddd}, J=8.1,7.0,1.1 \mathrm{~Hz}$, $1 \mathrm{H}), 6.21$ (br s, NH, 2H); ${ }^{13} \mathrm{C} \mathrm{NMR}\left(175 \mathrm{MHz}, \mathrm{CDCl}_{3}\right) \delta 197.8$ (C), $151.4(\mathrm{C}), 143.6(\mathrm{C}), 135.0(\mathrm{CH}), 134.5(\mathrm{CH}), 132.6(\mathrm{q}$, $\left.J_{\mathrm{C}-\mathrm{F}}=32.7 \mathrm{~Hz}, \mathrm{C}\right), 129.3(\mathrm{CH}), 125.3\left(\mathrm{q}, J_{\mathrm{C}-\mathrm{F}}=3.8 \mathrm{~Hz}, \mathrm{CH}\right)$, $123.9\left(\mathrm{q}, J_{\mathrm{C}-\mathrm{F}}=272.4 \mathrm{~Hz}, \mathrm{CH}\right), 117.4(\mathrm{C}), 117.3(\mathrm{CH}), 115.8$ (CH); IR (solid) 3487, 3069, 3040, 3021, 1672, 1590, 1575 $\mathrm{cm}^{-1}$; LRMS (ESI) $266\left(100,[\mathrm{M}+\mathrm{H}]^{+}\right)$; HRMS (ESI) calcd for $\mathrm{C}_{14} \mathrm{H}_{11} \mathrm{~F}_{3} \mathrm{NO}[\mathrm{M}+\mathrm{H}]^{+} 266.0787$; observed 266.0789.

(2-Aminophenyl)(2,3,4-trimethoxyphenyl)methanone $5 \mathrm{~h}$. Compound prepared according to method D. Purification by column chromatography for (10-25\% EtOAc/petrol) yielded (2-aminophenyl)(2,3,4-trimethoxyphenyl)methanone as a yellow crystalline solid (247 mg, $0.860 \mathrm{mmol}, 86 \%)$. Mp 123-125 ${ }^{\circ} \mathrm{C}$; ${ }^{1} \mathrm{H}$ NMR $\left(700 \mathrm{MHz}, \mathrm{CDCl}_{3}\right) \delta 7.30(\mathrm{dd}, J=8.1,1.5 \mathrm{~Hz}$, $1 \mathrm{H}), 7.21$ (ddd, $J=8.5,7.0,1.6 \mathrm{~Hz}, 1 \mathrm{H}), 6.95(\mathrm{~d}, J=8.5 \mathrm{~Hz}$, $1 \mathrm{H}), 6.68(\mathrm{~d}, J=8.5 \mathrm{~Hz}, 1 \mathrm{H}), 6.66(\mathrm{~d}, J=8.3,0.8 \mathrm{~Hz}, 1 \mathrm{H}), 6.51$ (ddd, $J=8.1,7.0,1.1 \mathrm{~Hz}, 1 \mathrm{H}), 6.35(\mathrm{br} \mathrm{s}, \mathrm{NH}, 2 \mathrm{H}), 3.88(\mathrm{~s}, 3 \mathrm{H})$, $3.88(\mathrm{~s}, 3 \mathrm{H}), 3.78(\mathrm{~s}, 3 \mathrm{H}) ;{ }^{13} \mathrm{C} \mathrm{NMR}\left(175 \mathrm{MHz}, \mathrm{CDCl}_{3}\right) \delta$ 198.0 (C), 155.1 (C), 151.6 (C), 151.1 (C), 142.1 (C), 134.9 (C), $134.6(\mathrm{CH}), 128.2(\mathrm{CH}), 123.7(\mathrm{CH}), 118.9(\mathrm{C}), 116.9$ $(\mathrm{CH}), 115.5(\mathrm{CH}), 106.9(\mathrm{CH}), 61.8\left(\mathrm{CH}_{3}\right), 61.1\left(\mathrm{CH}_{3}\right), 56.2$ $\left(\mathrm{CH}_{3}\right)$; IR (solid) 3500, 3084, 3065, 3029, 1670, 1593, 1571 $\mathrm{cm}^{-1}$; LRMS (ESI) $288\left(100,[\mathrm{M}+\mathrm{H}]^{+}\right)$; HRMS (ESI) calcd for $\mathrm{C}_{16} \mathrm{H}_{17} \mathrm{NO}_{4}[\mathrm{M}+\mathrm{H}]^{+} 288.1231$; observed 288.1235.

(2-Aminophenyl)(2-chlorophenyl)methanone $5 \mathrm{~g}$. Compound prepared according to method $\mathrm{D}$ with $0.36 \mathrm{mmol}$ of isopropyl (2-(2-chlorobenzoyl)phenyl)carbamate 3g. Purifica- 
tion by column chromotography for (10-25\% EtOAc/petrol) yielded (2-aminophenyl)(2-chlorophenyl)methanone as a yellow crystalline solid (75 mg, $0.33 \mathrm{mmol}, 91 \%)$. Mp 57-59 ${ }^{\circ} \mathrm{C}$; ${ }^{1} \mathrm{H}$ NMR $\left(700 \mathrm{MHz}, \mathrm{CDCl}_{3}\right) \delta 7.47-7.43(\mathrm{~m}, 1 \mathrm{H}), 7.38$ (ddd, $J=8.0,7.3,1.9 \mathrm{~Hz}, 1 \mathrm{H}), 7.34(\mathrm{td}, J=7.4,1.2 \mathrm{~Hz}, 1 \mathrm{H})$, $7.32-7.30(\mathrm{~m}, 1 \mathrm{H}), 7.28$ (ddd, $J=8.5,7.0,1.5 \mathrm{~Hz}, 1 \mathrm{H}), 7.17$ $(\mathrm{dd}, J=8.1,1.5 \mathrm{~Hz}, 1 \mathrm{H}), 6.72(\mathrm{~m}, 1 \mathrm{H}), 6.54(\mathrm{ddd}, J=8.1,7.0$, $1.1 \mathrm{~Hz}) 6.47(\mathrm{br} \mathrm{s}, \mathrm{NH}, 2 \mathrm{H}) ;{ }^{13} \mathrm{C} \mathrm{NMR}\left(175 \mathrm{MHz}, \mathrm{CDCl}_{3}\right) \delta$ $197.4(\mathrm{C}), 151.6(\mathrm{C}), 140.0(\mathrm{C}), 135.4(\mathrm{CH}), 134.8(\mathrm{CH})$, $130.8(\mathrm{C}), 130.5(\mathrm{CH}), 130.0(\mathrm{CH}), 128.6(\mathrm{CH}), 126.8(\mathrm{CH})$, $117.5(\mathrm{C}), 117.2(\mathrm{CH}), 115.9(\mathrm{CH})$; IR (solid) 3450, 3070, $3041,3029,1665,1591,1580 \mathrm{~cm}^{-1}$.

(2-Amino-5-bromophenyl)(2-chlorophenyl)methanone 8. To a solution of (2-aminophenyl)(2-chlorophenyl)methanone $5 \mathrm{~g}$ (75 mg, $0.33 \mathrm{mmol}, 1$ equiv) in dichloromethane $(10 \mathrm{~mL})$ was added $\mathrm{N}$-bromosuccinimide ( $59 \mathrm{mg}, 0.33 \mathrm{mmol}, 1$ equiv) at $0{ }^{\circ} \mathrm{C}$. The mixture was stirred for $1 \mathrm{~h}$ at this temperature. After completion of the reaction, the resulting mixture was quenched with sat. aq. $\mathrm{NaHCO}_{3}(20 \mathrm{~mL})$ and extracted into $\mathrm{DCM}(3 \times 15$ $\mathrm{mL})$. The combined organic layers were then dried $\left(\mathrm{MgSO}_{4}\right)$, and the solvent was removed in vacuo. Purification by column chromotography (10-25\% EtOAc/petrol) yielded (2-amino-5bromophenyl)(2-chlorophenyl)methanone as a yellow crystalline solid (92 mg, $0.30 \mathrm{mmol}, 90 \%) . \mathrm{Mp} 88-90{ }^{\circ} \mathrm{C}$; ${ }^{1} \mathrm{H}$ NMR $\left(700 \mathrm{MHz}, \mathrm{CDCl}_{3}\right) \delta 7.45(\mathrm{dd}, J=8.0,0.7 \mathrm{~Hz}, 1 \mathrm{H}), 7.40(\mathrm{td}, J=$ $7.8,1.7 \mathrm{~Hz}, 1 \mathrm{H}), 7.34(\mathrm{td}, J=7.5,1.1 \mathrm{~Hz}, 1 \mathrm{H}), 7.32(\mathrm{~d}, J=8.9$, $2.3 \mathrm{~Hz}, 1 \mathrm{H}), 7.29$ (dd $J=7.5,1.5 \mathrm{~Hz}, 1 \mathrm{H}), 7.25$ (d, $J=2.3 \mathrm{~Hz}$, $1 \mathrm{H}), 6.60(\mathrm{~d}, J=8.9 \mathrm{~Hz}, 1 \mathrm{H}), 6.52(\mathrm{br} \mathrm{s}, \mathrm{NH}, 2 \mathrm{H}) ;{ }^{13} \mathrm{C} \mathrm{NMR}$ $\left(175 \mathrm{MHz}, \mathrm{CDCl}_{3}\right) \delta 196.5$ (C), 150.5 (C), 139.1 (C), 138.0 (C), $136.3(\mathrm{C}), 131.0(\mathrm{C}), 130.2(\mathrm{CH}), 128.6(\mathrm{CH}), 127.0$ (CH), $119.1(\mathrm{C}), 118.7(\mathrm{CH}), 106.8(\mathrm{CH})$; IR (solid) 3350, 3041, 3022, 1627, $1570 \mathrm{~cm}^{-1}$.

Phenazepam. To a solution of (2-amino-5-bromophenyl)(2-chlorophenyl)methanone 8 (92 $\mathrm{mg}, 0.30 \mathrm{mmol}, 1$ equiv) in THF $(10 \mathrm{~mL})$ was added bromoacetyl bromide $(29 \mu \mathrm{L}, 0.33$ mmol, 1.1 equiv) at $0{ }^{\circ} \mathrm{C}$. The mixture was stirred for $1 \mathrm{~h}$ at this temperature. After this time, ammonium hydroxide $(1.5 \mathrm{~mL})$ in $\mathrm{EtOH}(2 \mathrm{~mL})$ was added dropwise, and the reaction was allowed to stir for $2 \mathrm{~h}$. After the completion of the reaction, the resulting mixture was quenched with sat. aq. $\mathrm{NaHCO}_{3}(20 \mathrm{~mL})$ and extracted into EtOAc $(3 \times 15 \mathrm{~mL})$. The combined organic layers were then dried $\left(\mathrm{MgSO}_{4}\right)$ and the solvent was removed in vacuo. Purification by column chromatography $(0-10 \% \mathrm{MeOH} /$ DCM) yielded (2-amino-5-bromophenyl)(2-chlorophenyl)methanone as a yellow crystalline solid $(89 \mathrm{mg}, 0.260 \mathrm{mmol}$, $85 \%) . \mathrm{Mp} 222-235^{\circ} \mathrm{C}$; ${ }^{1} \mathrm{H} \mathrm{NMR}\left(700 \mathrm{MHz}, \mathrm{CDCl}_{3}\right) \delta 8.61(\mathrm{br}$ s, NH, $1 \mathrm{H}), 7.57(\mathrm{dd}, J=8.6,2.3 \mathrm{~Hz}, 1 \mathrm{H}), 7.54-7.50(\mathrm{~m}, 1 \mathrm{H})$, $7.41-7.37(\mathrm{~m}, 3 \mathrm{H}), 7.20(\mathrm{~d}, J=2.2 \mathrm{~Hz}, 1 \mathrm{H}), 7.01(\mathrm{~d}, J=8.6 \mathrm{~Hz}$, $1 \mathrm{H}), 4.38(\mathrm{~s}, 2 \mathrm{H}) ;{ }^{13} \mathrm{C} \mathrm{NMR}\left(175 \mathrm{MHz}, \mathrm{CDCl}_{3}\right) \delta 170.9$ (C), 169.3 (C), 138.4 (C), 136.9 (C), 134.9 (C), 133.3 (C), 132.3 $(\mathrm{CH}), 131.2(\mathrm{CH}), 131.2(\mathrm{CH}), 130.4(\mathrm{CH}), 129.8(\mathrm{CH})$, $127.2(\mathrm{CH}), 122.7(\mathrm{C}), 116.9(\mathrm{CH}), 56.6\left(\mathrm{CH}_{2}\right)$; IR (solid) $3253,2900,1670,1505 \mathrm{~cm}^{-1}$.

Diisopropyl 1-(2-(tert-Butoxy)-2-oxoethyl)-2-(2-(4fluorobenzoyl)phenyl)hydrazine-1,2-dicarboxylate $4 a$. To a stirring solution of sodium hydride $(60 \%$ mineral oil dispersion, $40 \mathrm{mg}, 1.00 \mathrm{mmol}, 1.0$ equiv $)$ in anhydrous THF $(0.5$ $\mathrm{mL})$ was added dropwise a solution of diisopropyl 1-(2-(4fluorobenzoyl)phenyl)hydrazine-1,2-dicarboxylate $(0.5 \mathrm{mmol}$, 1 equiv) predissolved in anhydrous THF $(1 \mathrm{~mL})$ under an atmosphere of argon, and the reaction mixture was stirred for 5 $\mathrm{min}$. After this time, to the reaction mixture was added tert-butyl bromoacetate (162 $\mu \mathrm{L}, 1.10 \mathrm{mmol}, 1.1$ equiv) dropwise, predissolved in a $1.5 \mathrm{~mL}$ anhydrous THF. The reaction mixture was then stirred at $20{ }^{\circ} \mathrm{C}$ for $1 \mathrm{~h}$ and monitored via TLC till completion and poured over saturated aqueous $\mathrm{NH}_{4} \mathrm{Cl}(10 \mathrm{~mL})$. The resulting solution was extracted with EtOAc $(3 \times 15 \mathrm{~mL})$. The combined extracts were dried $\left(\mathrm{MgSO}_{4}\right)$ and filtered, and the solvent was evaporated in vacuo. Purification by column chromatography (10-50\% EtOAc/petrol) yielded diisopropyl 1-(2-(tert-butoxy)-2-oxoethyl)-2-(2-(4-fluorobenzoyl)phenyl)hydrazine-1,2-dicarboxylate as a yellow oil $(230 \mathrm{mg}, 0.445$ mmol, 89\%). ${ }^{1} \mathrm{H}$ NMR (400 MHz, $\left.\mathrm{CDCl}_{3}\right) \delta 7.80-7.88(\mathrm{~m}$, $3 \mathrm{H}), 7.49-7.55(\mathrm{~m}, 1 \mathrm{H}), 7.26(\mathrm{dd}, 2 \mathrm{H}, J=7.2,5.9 \mathrm{~Hz}), 7.09(\mathrm{t}$, $2 \mathrm{H}, J=8.2 \mathrm{~Hz}), 5.05(\mathrm{~m}, 1 \mathrm{H}), 4.74$ (septet, $1 \mathrm{H}, J=6.3 \mathrm{~Hz})$, 4.20-4.60 (m, 2H), 0.96-1.42 (m, 21H). ${ }^{13} \mathrm{C} \mathrm{NMR} \mathrm{(150} \mathrm{MHz,}$ $\left.\mathrm{CDCl}_{3}\right) \delta 193.8(\mathrm{C}), 167.7(\mathrm{C}), 166.6\left(\mathrm{~d}, J_{\mathrm{C}-\mathrm{F}}=253.4 \mathrm{~Hz}, \mathrm{CH}\right)$, 156.0 (C), 153.8 (C), 139.0 (C), 134.3 (C), $133.3(\mathrm{CH}), 131.8$ $(\mathrm{CH}), 131.6(\mathrm{CH}), 128.9(\mathrm{CH}), 128.2(\mathrm{CH}), 127.0(\mathrm{CH})$, $126.3(\mathrm{CH}), 125.9(\mathrm{CH}), 115.5\left(\mathrm{~d}, J_{\mathrm{C}-\mathrm{F}}=22.0 \mathrm{~Hz}, \mathrm{CH}\right), 81.3$ (C), $71.0(\mathrm{CH}), 52.5(\mathrm{CH}), 28.2(\mathrm{CH}), 22.2(\mathrm{CH})$; IR (thin film) $3341,2989,2967,1743,1620,1583,1523 \mathrm{~cm}^{-1}$; LRMS (ESI) $517\left(100,[\mathrm{M}+\mathrm{H}]^{+}\right)$, HRMS (ESI) calcd for $\mathrm{C}_{27} \mathrm{H}_{34} \mathrm{FN}_{2} \mathrm{O}_{7}[\mathrm{M}+\mathrm{H}]^{+}$517.2342; observed 517.2348.

\section{ASSOCIATED CONTENT}

\section{Supporting Information}

The Supporting Information is available free of charge at https://pubs.acs.org/doi/10.1021/acsomega.9b03417.

Copies of characterization data $\left({ }^{1} \mathrm{H}\right.$ and ${ }^{13} \mathrm{C}$ NMR $)$ of all featured compounds; and detailed experimental procedure, NMR $\left({ }^{1} \mathrm{H}\right.$ and $\left.{ }^{13} \mathrm{C}\right)$, IR, LRMS (ESI), and HRMS (ESI) of all featured compounds (PDF)

\section{AUTHOR INFORMATION}

\section{Corresponding Author}

*E-mail: v.chudasama@ucl.ac.uk.

ORCID $\odot$

Vijay Chudasama: 0000-0002-8876-3285

\section{Author Contributions}

The manuscript was written through contributions of all authors. All authors have given approval to the final version of the manuscript. N.A. and A.S. contributed equally.

Notes

The authors declare no competing financial interest.

\section{ACKNOWLEDGMENTS}

The authors gratefully acknowledge the UCL Graduate School for funding A.S. The authors also acknowledge the UCL Chemistry Mass Spectrometry Facility (Dr. K. Karu/Dr. X. Yang) and the EPSRC U.K. National MS Facility (Swansea).

\section{REFERENCES}

(1) Liou, J.-P.; Chang, C.-W.; Song, J.-S.; Yang, Y.-N.; Yeh, C.-F.; Tseng, H.-Y.; Lo, Y.-K.; Chang, Y.-L.; Chang, C.-M.; Hsieh, H.-P. Synthesis and Structure-Activity Relationship of 2-Aminobenzophenone Derivatives as Antimitotic Agents. J. Med. Chem. 2002, 45, 25562562.

(2) Singh, R. K.; Prasad, D. N.; Bhardwaj, T. R. Design, Synthesis and Evaluation of Aminobenzophenone Derivatives Containing Nitrogen Mustard Moiety as Potential Central Nervous System Antitumor Agent. Med. Chem. Res. 2013, 22, 5901-5911.

(3) Castellano, S.; Taliani, S.; Viviano, M.; Milite, C.; Da Pozzo, E.; Costa, B.; Barresi, E.; Bruno, A.; Cosconati, S.; Marinelli, L.; Greco, G.; Novellino, E.; Sbardella, G.; Da Settimo, F.; Martini, C. Structure- 
Activity Relationship Refinement and Further Assessment of 4Phenylquinazoline-2-Carboxamide Translocator Protein Ligands as Antiproliferative Agents in Human Glioblastoma Tumors. J. Med. Chem. 2014, 57, 2413-2428.

(4) Singh, R. K.; Devi, S.; Prasad, D. N. Synthesis, Physicochemical and Biological Evaluation of 2-Amino-5-Chlorobenzophenone Derivatives as Potent Skeletal Muscle Relaxants. Arab. J. Chem. 2015, 8, 307-312.

(5) Wu, H.; Zhang, Z.; Liu, Q.; Liu, T.; Ma, N.; Zhang, G. Syntheses of Acridones via Copper(II)-Mediated Relay Reactions from o-Aminoacetophenones and Arylboronic Acids. Org. Lett. 2018, 20, 2897-2901.

(6) Shirini, F.; Akbari-Dadamahaleh, S.; Mohammad-Khah, A. Rice Husk Ash Supported FeCl2.2H2O: A Mild and Highly Efficient Heterogeneous Catalyst for the Synthesis of Polysubstituted Quinolines by Friedländer Heteroannulation. Chinese J. Catal. 2013, 34, 2200-2208.

(7) Zakerinasab, B.; Nasseri, M. A.; Kamali, F. Efficient Procedure for the Synthesis of Quinoline Derivatives by NbCl5.PEG and $\mathrm{NbCl5}$ in Glycerol as Green Solvent. Iran. Chem. Commun. 2015, 3, 335-347.

(8) Reddy, B. P.; Iniyavan, P.; Sarveswari, S.; Vijayakumar, V. Nickel Oxide Nanoparticles Catalyzed Synthesis of Poly-Substituted Quinolines via Friedlander Hetero-Annulation Reaction. Chinese Chem. Lett. 2014, 25, 1595-1600.

(9) Karnakar, K.; Kumar, A. V.; Murthy, S. N.; Ramesh, K.; Nageswar, Y. V. D. Recyclable Graphite Oxide Promoted Efficient Synthesis of 2Phenyl Quinazoline Derivatives in the Presence of TBHP as an Oxidant. Tetrahedron Lett. 2012, 53, 4613-4617.

(10) Zhang, J.; Zhu, D.; Yu, C.; Wan, C.; Wang, Z. A Simple and Efficient Approach to the Synthesis of 2-Phenylquinazolines via Sp3 CH Functionalization. Org. Lett. 2010, 12, 2841-2843.

(11) Dabiri, M.; Salehi, P.; Bahramnejad, M. Ecofriendly and Efficient One-Pot Procedure for the Synthesis of Quinazoline Derivatives Catalyzed by an Acidic Ionic Liquid Under Aerobic Oxidation Conditions. Synth. Commun. 2010, 40, 3214-3225.

(12) Park, K. K.; Lee, J. J. Facile Synthesis of 4-Phenylquinolin-2(1H)One Derivatives from N-Acyl-o-Aminobenzophenones. Tetrahedron 2004, 60, 2993-2999.

(13) Shobha, D.; Chari, M. A.; Mukkanti, K.; Kim, S. Y. Synthesis and Anti-Neuroinflammatory Activity Studies of Substituted 3,4-Dihydroquinoxalin-2-Amine Derivatives. Tetrahedron Lett. 2012, 53, 26752679.

(14) Zhang, Z.; Kodumuru, V.; Sviridov, S.; Liu, S.; Chafeev, M.; Chowdhury, S.; Chakka, N.; Sun, J.; Gauthier, S. J.; Mattice, M.; Ratkay, L. G.; Kwan, R.; Thompson, J.; Cutts, A. B.; Fu, J.; Kamboj, R.; Goldberg, Y. P.; Cadieux, J. A. Discovery of Benzylisothioureas as Potent Divalent Metal Transporter 1 (DMT1) Inhibitors. Bioorg. Med. Chem. Lett. 2012, 22, 5108-5113.

(15) Chiarini, M.; Del Vecchio, L.; Mainelli, F.; Rossi, L.; Arcadi, A. Synthesis of 3-Substituted 2,1-Benzisoxazoles by the Oxidative Cyclization of 2-Aminoacylbenzenes with Oxone. Synthesis 2016, 48, 3017-3030.

(16) Mochalov, S. S.; Khasanov, M. I.; Fedotov, A. N.; Trofimova, E. V. 2H-Indazoles from 2-Aminobenzophenones. Chem. Heterocycl. Compd. 2008, 44, 229-230.

(17) Paul, N.; Muthusubramanian, S. Reaction of Diphenacylanilines with 2-Aminobenzophenone: An Abnormal Friedlander Reaction Yielding Indoles. Synth. Commun. 2013, 43, 1200-1209.

(18) Sbei, N.; Batanero, B.; Barba, F.; Haouas, B.; Benkhoud, M. L.; Barba, I. Facile Preparation of 3-Substituted 2-Quinazolinones via Electrogenerated Base. Tetrahedron 2018, 74, 2068-2072.

(19) Park, K. K.; Lee, J. H. Efficient Synthesis of 2,3-Disubstituted Benzo[b]Thiophenes Starting from 2-Aminobenzophenone. Bull. Korean Chem. Soc. 2008, 29, 2502-2504.

(20) Acharya, B. P.; Rao, Y. R. Synthesis of Unsymmetrically Substituted 6,12-Diaryldibenzo[b,f] $[1,5]$ Diazocines and Their Precursor Schiff Bases. Synthesis 1986, 324-326.

(21) Reddy, D. R.; Ballante, F.; Zhou, N. J.; Marshall, G. R. Design and Synthesis of Benzodiazepine Analogs as Isoform-Selective Human Lysine Deacetylase Inhibitors. Eur. J. Med. Chem. 2017, 127, 531-553.
(22) Cheng, P.; Zhang, Q.; Ma, Y. B.; Jiang, Z. Y.; Zhang, X. M.; Zhang, F. X.; Chen, J. J. Synthesis and in Vitro Anti-Hepatitis B Virus Activities of 4-Aryl-6-Chloro-Quinolin-2-One and 5-Aryl-7-Chloro1,4-Benzodiazepine Derivatives. Bioorg. Med. Chem. Lett 2008, 18, 3787-3789.

(23) Huang, Y.; Khoury, K.; Chanas, T.; Dömling, A. Multicomponent Synthesis of Diverse 1,4-Benzodiazepine Scaffolds. Org. Lett. 2012, 14, 5916-5919.

(24) Fier, P. S.; Whittaker, A. M. An Atom-Economical Method to Prepare Enantiopure Benzodiazepines with N-Carboxyanhydrides. Org. Lett. 2017, 19, 1454-1457.

(25) Cortez-Maya, S.; Cortes Cortes, E.; Hernández-Ortega, S.; Apan, T. R.; Martínez-García, M. Synthesis of 2-Aminobenzophenone Derivatives and Their Anticancer Activity. Synth. Commun. 2012, 42, $46-54$.

(26) Tran, P. H.; Phung, H. Q.; Hansen, P. E.; Tran, H. N.; Le, T. N. Efficient Friedel-Crafts Benzoylation of Aniline Derivatives with 4Fluorobenzoyl Chloride Using Copper Triflate in the Synthesis of Aminobenzophenones. Synth. Commun. 2016, 46, 893-901.

(27) Yan, Y.; Wang, Z. Metal-Free Intramolecular Oxidative Decarboxylative Amination of Primary $\alpha$-Amino Acids with Product Selectivity. Chem. Commun. 2011, 47, 9513-9515.

(28) Chen, J.; Ye, L.; Su, W. Palladium-Catalyzed Direct Addition of Arylboronic Acids to 2-Aminobenzonitrile Derivatives: Synthesis, Biological Evaluation and in Silico Analysis of 2-Aminobenzophenones, 7-Benzoyl-2-Oxoindolines, and 7-Benzoylindoles. Org. Biomol. Chem. 2014, 12, 8204-8211.

(29) Chen, J.; Li, J.; Su, W. Palladium-Catalyzed Direct Addition of 2Aminobenzonitriles to Sodium Arylsulfinates: Synthesis of o-Aminobenzophenones. Molecules 2014, 19, 6439-6449.

(30) Szabó, F.; Simkó, D.; Novák, Z. A One-Pot Process for Palladium Catalyzed Direct C-H Acylation of Anilines in Water Using a Removable Ortho Directing Group. RSC Adv. 2014, 4, 3883-3886.

(31) Pintori, D. G.; Greaney, M. F. Insertion of Benzene Rings into the Amide Bond: One-Step Synthesis of Acridines and Acridones from Aryl Amides. Org. Lett. 2010, 12, 168-171.

(32) Wright, A. C.; Haley, C. K.; Lapointe, G.; Stoltz, B. M. Synthesis of Aryl Ketoamides via Aryne Insertion into Imides. Org. Lett. 2016, 18, 2793-2795.

(33) Shamsabadi, A.; Chudasama, V. An Overview of the Synthesis of Acyl Hydrazides from Aldehydes and Reactions of the Products Thereof. Org. Biomol. Chem. 2017, 15, 17-33.

(34) Maruani, A.; Lee, M. T. W.; Watkins, G.; Akhbar, A. R.; Baggs, H.; Shamsabadi, A.; Richards, D. A.; Chudasama, V. A Facile, One-Pot Procedure for the Conversion of Aromatic Aldehydes to Esters, as well as Thioesters and Amides, via Acyl Hydrazide Intermediates. RSC Adv. 2016, 6, 3372-3376.

(35) Akhbar, A. R.; Chudasama, V.; Fitzmaurice, R. J.; Powell, L.; Caddick, S. Acyl Hydrazides as Acyl Donors for the Synthesis of Diaryl and Aryl Alkyl Ketones. Chem. Commun. 2014, 50, 743-746.

(36) Shamsabadi, A.; Ren, J.; Chudasama, V. Enabling the Facile Conversion of Acyl Hydrazides into: N-Acyl Carbamates via MetalFree Ionic-Based Rupture of the N-N Linkage. RSC Adv. 2017, 7, 27608-27611.

(37) Shamsabadi, A.; Chudasama, V. A Facile Route to $1 \mathrm{H}$ - and $2 \mathrm{H}$ Indazoles from Readily Accessible Acyl Hydrazides by Exploiting a Novel Aryne-Based Molecular Rearrangement. Chem. Commun. 2018, 54, 11180-11183.

(38) Sugimoto, O.; Arakaki, T.; Kamio, H.; Tanji, K. The Use of a Mitsunobu Reagent for the Formation of Heterocycles: A Simple Method for the Preparation of 3-Alkyl-5-Aryl-1,3,4-Oxadiazol-2(3H)Ones from Carboxylic Acids. Chem. Commun. 2014, 50, 7314-7317.

(39) Papadopoulos, G. N.; Kokotos, C. G. Photoorganocatalytic OnePot Synthesis of Hydroxamic Acids from Aldehydes. Chem. Eur. J. 2016, 22, 6964-6967.

(40) Kim, Y. J.; Lee, D. Use of N-N Bond Stereodynamics in RingClosing Metathesis to Form Medium-Sized Rings and Macrocycles. Org. Lett. 2004, 6, 4351-4353. 
(41) Magnus, P.; Garizi, N.; Seibert, K. A.; Ornholt, A. Synthesis of Carbamates from Diethoxycarbonyl Hydrazine Derivatives by E1cB Eliminative Cleavage of the N-N'-Bond Rather than Reduction. Org. Lett. 2009, 11, 5646-5648.

(42) Takemoto, Y.; Matsumoto, T.; Ito, Y.; Terashima, S. An Expeditious Synthesis of (3S,4S)-Statine and (3S,4S)- Cyclohexylstatine. Tetrahderon Lett. 1990, 31, 217-218.

(43) Gaboardi, M.; Castaldi, G.; Castaldi, M. Process and Intermediates for the Preparation of Linagliptin. WO Patent WO0675392015.

(44) Chee, G.-L. Selective Deprotection of Isopropyl Esters, Carbamates and Carbonates with Aluminium Chloride. Synlett 2001, $1593-1595$.

(45) Yang, Y.; Xu, Y.; Jones, C. R. A Ring Expansion Route to Benzofused N-Heterocycles Through Aryne Insertion into 1,3-DiazaHeterocycles. Eur. J. Org. Chem. 2019, 5196-5200.

(46) Lukashov, O. I.; Kazakov, P. V.; Mirzabekova, N. S. Optimization of Phenazepam Production Technology. Pharm. Chem. J. 2018, 52, 641-643.

(47) Andronati, S. A.; Makan, S. Y.; Neshchadin, D. P.; Yakubovskaya, L. N.; Sava, V. M.; Andronati, K. S. Bioaccessibility of Cinazepam Introduced as Inclusion Complex with Beta-Cyclodextrin. Pharm. Chem. J. 1998, 32, 513-515. 